IGC-08/10-2

\title{
Dilaton Gravity, Poisson Sigma Models and Loop Quantum Gravity
}

\author{
Martin Bojowald and Juan D. Reyest \\ Institute for Gravitation and the Cosmos, \\ The Pennsylvania State University, \\ 104 Davey Lab, University Park, PA 16802, USA
}

\begin{abstract}
Spherically symmetric gravity in Ashtekar variables coupled to Yang-Mills theory in two dimensions and its relation to dilaton gravity and Poisson sigma models are discussed. After introducing its loop quantization, quantum corrections for inverse triad components are shown to provide a consistent deformation without anomalies. The relation to Poisson sigma models provides a covariant action principle of the quantum corrected theory with effective couplings. Results are also used to provide loop quantizations of spherically symmetric models in arbitrary $D$ space-time dimensions.
\end{abstract}

\section{Introduction}

Approaches to quantum gravity imply characteristic corrections to classical general relativity, by which their viability can be tested and which may eventually give rise to observable indications. Distinct approaches often lead to corrections of very different forms, but this is not necessarily contradictory: Formulations are usually difficult to compare directly which may shroud similar implications of different-looking terms, especially if canonical formulations on the one hand and Lagrangian ones on the other are used. In particular, it is not always straightforward to derive action principles corresponding to quantum corrected Hamiltonian formulations.

In the canonical approach underlying loop quantum gravity [1, 2, 3], characteristic corrections in equations of motion arise from three sources: corrections of inverse components of the densitized triad which is used instead of a spatial metric [4, 5], higher order corrections of connection components (due to the use of holonomies [6]), and genuine quantum effects due to the back-reaction of higher moments of a state on its expectation values. The latter is generic for any interacting quantum system, where it leads, e.g., to effective

*e-mail address: bojowald@gravity.psu.edu

${ }^{\dagger}$ e-mail address: jdr234@psu.edu 
potentials; for a canonical treatment see [7, 8]. The first two sources of corrections are characteristic of loop quantum gravity and directly related to the underlying discreteness of its spatial geometry.

In homogeneous models of loop quantum cosmology [9], these corrections have been analyzed in quite some detail. In such settings, however, consistency conditions (such as anomaly freedom) trivialize which leaves much freedom in the form of corrections and a high level of ambiguity in any effective action which might correspond to the corrected equations of motion. In this article, we thus consider spherically symmetric models [10] as an intermediate step where the consistency issue is non-trivial but calculations are still manageable. As it turns out, correction terms in effective actions are then determined much more uniquely.

We will exploit the fact that spherically symmetric gravity, or more generally 2-dimensional dilaton gravity [11, 12], can be formulated as a Poisson sigma model (PSM) which algebraically has a very rigid structure: a consistent deformation (such as an anomaly-free quantization) of a PSM can only be another PSM [13]. There is thus a clear way of interpreting any consistent way of introducing quantum corrections. Applying this to loop quantum gravity will then provide a covariant interpretation of corrections in the form of a corrected Poisson structure of the target manifold, or an effective dilaton potential. We also note that PSMs play a role as models of string theory, such that an analysis along the lines followed here may shed light on the relation between loop quantum gravity and string theory.

The specific correction analyzed here is only the inverse triad term, which is one of the terms directly linked to the discreteness, while, for now, we ignore holonomy corrections and quantum back-reaction. We will show that this can be implemented as a consistent deformation under certain conditions on the phase-space dependence of the correction, and then relate it to changes in the underlying Poisson structure of the PSM formulation. To that end, we first review the different underlying classical formulations in Sec. 2 and derive an explicit canonical transformation to relate them in Sec. 3. After introducing quantum corrections at the level of the loop formulation, as it is reviewed in Sec. 5 in nearly selfcontained form, we will translate this back to the PSM formulation in Sec. 6. The analysis will be done in the presence of a Yang-Mills source which can, as detailed in Sec. 4, be added as an extension of the original PSM.

\section{Formulations of gravity in two dimensions}

In two dimensions the analog of the Einstein-Hilbert action in vacuum is trivial, but the presence of extra fields gives rise to interesting models. Such fields may, for instance, arise after dimensional reduction of a field theory in four or higher dimensions. In the case of $2 \mathrm{~d}$ gravity this leads to the presence of the dilaton field. Most of the studied gravity theories in two dimensions can be described by the so-called Generalized Dilaton Gravity action

$$
S[\mathbf{g}, \Phi]=\frac{1}{2} \int_{M} d^{2} x \sqrt{-\mathbf{g}}\left(\mathbf{F}(\Phi) R(\mathbf{g})+\mathbf{U}(\Phi) \mathbf{g}^{\mu \nu} \partial_{\mu} \Phi \partial_{\nu} \Phi+\mathbf{V}(\Phi)\right)
$$


which is the most general diffeomorphism invariant action giving second order differential equations for a metric $\mathbf{g}$ and a scalar dilaton field $\Phi$ on a two-dimensional manifold $M$; for a comprehensive review see [12]. Here, F, U and $\mathbf{V}$ are three functions parameterizing different models (which should be sufficiently well behaved, and $\mathbf{F}$ being invertible).

\subsection{Poisson Sigma Models}

Poisson sigma models are a more general and unifying structure encompassing and generalising 2d gravity theories as well as 2d Yang-Mills theories [14, 15, 16, 11]. These are topological two-dimensional field theories which encode all the content of a particular model in a single Poisson tensor $\mathcal{P}$ defined on an $n$-dimensional target manifold $N$ (in local coordinates $\left.\mathbf{X}^{i}, \mathcal{P}=\frac{1}{2} \mathcal{P}^{i j}(\mathbf{X}) \frac{\partial}{\partial \mathbf{X}^{i}} \wedge \frac{\partial}{\partial \mathbf{X}^{j}}\right)$.

For a given Poisson tensor, the corresponding PSM action has the form

$$
S_{\mathrm{PSM}}=\int_{M} \mathbf{A}_{i} \wedge d \mathbf{X}^{i}+\frac{1}{2} \mathcal{P}^{i j} \mathbf{A}_{i} \wedge \mathbf{A}_{j}
$$

or, written explicitly with coordinates $x^{\mu}$ on $M$,

$$
S_{\mathrm{PSM}}=\int_{M} d x^{\mu} \wedge d x^{\nu}\left[\mathbf{A}_{i \mu}(x) \frac{\partial \mathbf{X}^{i}}{\partial x^{\nu}}(x)+\frac{1}{2} \mathcal{P}^{i j}(\mathbf{X}(x)) \mathbf{A}_{i \mu}(x) \mathbf{A}_{j \nu}(x)\right] .
$$

The dynamical fields are $\mathbf{X}^{i}\left(x^{\mu}\right)$, which parameterize a map $\mathcal{X}: M \rightarrow N$ from the twodimensional spacetime manifold $M$ to the target Poisson manifold $N$, as well as $\mathbf{A}_{i}=$ $\mathbf{A}_{i \mu} d x^{\mu}$, a one-form on $M$ taking values in $\mathcal{X}^{*}\left(T^{*} N\right)$.

More abstractly, the pair $\left(\mathbf{X}^{i}, \mathbf{A}_{i}\right)$ defines a vector bundle morphism $T M \rightarrow T^{*} N$ with base map $\mathcal{X}$, so that the action may be viewed as a functional of vector bundle morphisms. The equations of motion of the PSM may be shown to require these morphisms to preserve the standard Lie algebroid structures 1 on $T M$ and $T^{*} N$ : solutions to PSMs are Lie algebroid morphisms, and gauge symmetries are related to homotopies of morphisms [17].

The key step in establishing $2 d$ gravity as a PSM is the reformulation of (1) in first order form by using Einstein-Cartan variables: dyads and the spin connection instead of the metric. First, using the field redefinition $\phi=\mathbf{F}(\Phi)$ and replacing $\mathbf{U}$ by $\mathbf{U} / \mathbf{F}^{\prime 2}$, the coefficient $\mathbf{F}$ for the curvature term may always be assumed to be the identity function. The kinetic term can be eliminated by means of a conformal transformation $g:=\Omega^{2}(\phi) \mathbf{g}$, with

$$
\Omega(\phi)=\exp \left(\int^{\phi} \frac{\mathbf{U}(z)}{2} d z+\text { const }\right)
$$

and $V=-\mathbf{V}(\phi) / \Omega^{2}(\phi)$. The action

$$
S=\frac{1}{2} \int_{M} d^{2} x \sqrt{-g}(\phi R-V(\phi))
$$

\footnotetext{
${ }^{1} \mathrm{~A}$ Lie algrebroid is essentially a fiber bundle with a Lie bracket defined on its sections as well as an anchor map from the bundle to the tangent bundle over the same base manifold. A Lie algebra is a Lie algebroid whose base manifold is a single point.
} 
can then be expressed in first order form using dyads $e_{\mu}^{a} d x^{\mu}$ and connection one-forms $\omega_{\mu} \stackrel{a}{b} d x^{\mu}$ :

$$
S=-\int_{M} \phi d \omega+\frac{1}{2} V(\phi) \varepsilon+X_{a} D e^{a} .
$$

Here, we have used the two-dimensional identity $R \varepsilon=-2 d \omega$ where $\varepsilon$ is the two-dimensional volume form and $\omega$ is defined by $\omega_{b}^{a}=\omega \varepsilon_{b}^{a}$, with $\varepsilon_{b}^{a}$ being the single generator of the Lorentz gauge algebra $\mathfrak{s o}(1,1)$. Lagrange multipliers $X^{a}$ are introduced to enforce the condition of torsion freedom; see App. B for theories with torsion.

\subsubsection{Equations of motion}

Variation of (3) with respect to $\phi, \omega, X_{a}$ and $e^{a}$ respectively gives the equations of motion

$$
\begin{gathered}
d \omega+\frac{1}{2} V^{\prime}(\phi) \varepsilon=0 \\
d \phi+X_{a} \varepsilon_{b}^{a} e^{b}=0 \\
D e^{a}=d e^{a}+\varepsilon^{a}{ }_{b} \omega \wedge e^{b}=0 \\
\frac{1}{2} V(\phi) \varepsilon_{a b} e^{b}+d X_{a}+\varepsilon_{a b} X^{b} \omega=0 .
\end{gathered}
$$

It is convenient to introduce a light cone basis $e^{ \pm}$for dyads, so that $g_{\mu \nu}=2 e_{(\mu}^{+} e_{\nu)}^{-}$and raising and lowering indices is accomplished by replacing a lower $+(-)$ by an upper $-(+)$ and vice versa. Solving the condition (6) for torsion freedom with coordinates $(t, x)$ on $M$ and a light cone basis, we get the spin connection in terms of the dyad:

$$
\begin{aligned}
\omega_{x} & =\frac{\left(e_{x}^{-} e_{x}^{+}\right)^{\cdot}-\left(e_{t}^{-} e_{x}^{+}+e_{t}^{+} e_{x}^{-}\right)^{\prime}+e_{t}^{-} e_{x}^{+\prime}+e_{t}^{+} e_{x}^{-\prime}}{e_{t}^{+} e_{x}^{-}-e_{x}^{+} e_{t}^{-}} \\
\omega_{t} & =\frac{\dot{e}_{x}^{-} e_{t}^{+}+\dot{e}_{x}^{+} e_{t}^{-}-\left(e_{t}^{-} e_{t}^{+}\right)^{\prime}}{e_{t}^{+} e_{x}^{-}-e_{x}^{+} e_{t}^{-}}
\end{aligned}
$$

From Eq. (5) we obtain

$$
X^{\mp}=\frac{\phi^{\prime} e_{t}^{\mp}-\dot{\phi} e_{x}^{\mp}}{e_{t}^{+} e_{x}^{-}-e_{x}^{+} e_{t}^{-}} .
$$

These equations will be useful below.

\subsubsection{Spherically Symmetric Gravity as a Poisson sigma model}

To relate dilaton gravity and PSMs to spherically reduced gravity in four dimensions, we start from the Einstein-Hilbert action

$$
S_{\mathrm{EH}}=\frac{1}{16 \pi G} \int_{M \times S^{D-2}} d^{D} x{\sqrt{-{ }^{D} g}}^{D} R
$$


in $D$ dimensions and insert the ansatz

$$
d s^{2}=\mathbf{g}_{\mu \nu}\left(x^{\mu}\right) d x^{\mu} d x^{\nu}+\Phi^{2}\left(x^{\mu}\right) d \Omega_{S^{D-2}}^{2}
$$

for a spherically symmetric metric with $\mathbf{g}_{\mu \nu}$ a metric of signature $(-,+)$ on the twodimensional spacetime $M$, and $d \Omega_{S^{D-2}}^{2}$ the area element of the $(D-2)$-sphere $S^{D-2}$ (for the 2-sphere $d \Omega^{2}=d \vartheta^{2}+\sin ^{2} \vartheta d \varphi^{2}$ ). After integration of the angular variables (see e.g. App. B of [18] or App. C of [19]), the reduced 2d dilaton action is

$S=\frac{\mathcal{O}_{D-2}}{16 \pi G} \int_{M} d^{2} x \sqrt{-\mathbf{g}}\left[\Phi^{D-2} \mathbf{R}(\mathbf{g})+(D-2)(D-3) \Phi^{D-4} \mathbf{g}^{\mu \nu} \partial_{\mu} \Phi \partial_{\nu} \Phi+(D-2)(D-3) \Phi^{D-4}\right]$.

with $\mathcal{O}_{D-2}$ the volume of $S^{D-2}$.

Defining

$$
\phi:=\Phi^{D-2} \quad, \quad g_{\mu \nu}:=\phi^{\frac{D-3}{D-2}} \mathbf{g}_{\mu \nu} \quad, \quad V(\phi):=-(D-2)(D-3) \phi^{-1 / D-2}
$$

and introducing Lagrange multipliers $X_{a}$ as before to implement torsion-freedom, this action is seen to be of the form (3):

$$
S=-\frac{\mathcal{O}_{D-2}}{8 \pi G} \int_{M} X_{a} d e^{a}+X_{a} \varepsilon_{b}^{a} \omega \wedge e^{b}+\phi d \omega+\frac{1}{2} V(\phi) \varepsilon .
$$

Further, integrating by parts and discarding boundary terms we have

$$
S=-\frac{\mathcal{O}_{D-2}}{8 \pi G} \int_{M} e^{a} \wedge d X_{a}+\omega \wedge d \phi+X_{a} \varepsilon_{b}^{a} \omega \wedge e^{b}+\frac{1}{2} V(\phi) \varepsilon .
$$

If we collect the zero- and one-forms appearing in the last equation into the multiplets

$$
\begin{aligned}
& \left(\mathbf{X}^{i}\right):=\left(X^{a}, \phi\right)=\left(X^{-}, X^{+}, \phi\right) \\
& \left(\mathbf{A}_{i}\right):=\left(e_{a}, \omega\right)=\left(e^{+}, e^{-}, \omega\right)
\end{aligned}
$$

and use the Poisson bivector

$$
\mathcal{P}^{i j}=\left(\begin{array}{ccc}
0 & -V / 2 & -X^{-} \\
V / 2 & 0 & X^{+} \\
X^{-} & -X^{+} & 0
\end{array}\right),
$$

the action finally takes the form of a Poisson sigma model:

$$
S=-\frac{\mathcal{O}_{D-2}}{8 \pi G} \int_{M} \mathbf{A}_{i} \wedge d \mathbf{X}^{i}+\frac{1}{2} \mathcal{P}^{i j} \mathbf{A}_{i} \wedge \mathbf{A}_{j}
$$

with a three-dimensional target space, and $i \in\{-,+, 3\}$.

From here on we specialize to four dimensions for which

$$
\phi:=\Phi^{2} \quad, \quad g_{\mu \nu}:=\sqrt{\phi} \mathbf{g}_{\mu \nu} \quad, \quad V(\phi):=-\frac{2}{\sqrt{\phi}}
$$




\subsubsection{Canonical Form}

The action for a Poisson sigma model is already in first order form. Using coordinates $(t, x)$ on $M$, (14), specialized to four dimensions, reads:

$$
S=\frac{1}{2 G} \int d t \int d x\left[A_{i} \dot{\mathbf{X}}^{i}-\Lambda_{i}\left(\mathbf{X}^{i \prime}+\mathcal{P}^{i j} A_{j}\right)\right]
$$

with

$$
A_{i}:=\mathbf{A}_{x i} \quad, \quad \Lambda_{i}:=\mathbf{A}_{t i} \quad, \quad \dot{\mathbf{X}}:=\partial_{t} \mathbf{X} \quad, \quad \mathbf{X}^{\prime}:=\partial_{x} \mathbf{X} .
$$

The canonically conjugate variables are thus $\mathbf{X}^{i}$ and $A_{i}$ with

$$
\left\{\mathbf{X}^{i}(x), A_{i}(y)\right\}=2 G \delta_{j}^{i} \delta(x-y),
$$

subject to the total constraint

$$
\int d x \Lambda_{i} \tilde{C}^{i} \approx 0
$$

with Lagrange multipliers $\Lambda_{i}$ and

$$
\tilde{C}^{i}:=\frac{1}{2 G}\left(\mathbf{X}^{i \prime}+\mathcal{P}^{i j} A_{j}\right) .
$$

These constraints form the first class algebra

$$
\left\{C\left[\Lambda_{i}\right], C\left[K_{j}\right]\right\}=-\frac{1}{2 G} C\left[\Lambda_{i} K_{l} \partial_{k} \mathcal{P}^{i l}\right] .
$$

For a linear Poisson tensor, this is an algebra with structure constants, equivalent to the Gauss constraint of a gauge theory with structure constants $\partial_{k} \mathcal{P}^{i j}$. For non-linear Poisson tensors, on the other hand, the system has structure functions. The Lie algebroid formulation of Poisson sigma models, alluded to above, provides an interesting perspective on systems with structure functions whose constraints generate the symmetries of a Lie algebroid rather than a local Lie algebra. Similar interpretations exist for a large class of algebroid Yang-Mills theories [20] or Dirac sigma models [21].

The action (3) is space-time diffeomorphism and $\mathrm{SO}(1,1)$-gauge invariant. In particular, $\tilde{C}^{3}$ is the canonical generator of local gauge transformations, and the spatial diffeomorphism constraint is the combination

$$
\tilde{D}:=A_{i} \tilde{C}^{i}=A_{i} \mathbf{X}^{i \prime} .
$$

For the relation to variables underlying the loop formulation, it will be convenient to introduce the $\mathrm{SO}(1,1)$ invariant quantities

$$
X^{2}:=X^{-} X^{+} \quad, \quad e^{2}:=e_{x}^{-} e_{x}^{+}
$$

as well as gauge angles $\alpha$ and $\beta$ by

$$
X^{ \pm}=X \exp ( \pm \beta) \quad, \quad e_{x}^{ \pm}=e \exp ( \pm \alpha) .
$$


The angles are well-defined as long as $X \neq 0$ and $e \neq 0$, which is the case except at horizons. In what follows, we analyze the local constraint algebra such that global problems of this transformation of variables do not play a role. (As expected for an Abelian gauge transformation, $C^{3}[\Lambda]$ then generates $\alpha \rightarrow \alpha-\Lambda, \beta \rightarrow \beta-\Lambda, \omega_{x} \rightarrow \omega_{x}+\Lambda^{\prime}$. In the regions where our change of variables is valid one can even Abelianize the full constraint system; see [11].)

The symplectic structure $\Omega$ in the new variables becomes

$$
\begin{aligned}
\Omega & =\frac{1}{2 G} \int d x \delta A_{i} \wedge \delta \mathbf{X}^{i} \\
& =\frac{1}{2 G} \int d x \delta(e \exp \alpha) \wedge \delta(X \exp -\beta)+\delta(e \exp -\alpha) \wedge \delta(X \exp \beta)+\delta \omega_{x} \wedge \delta \phi \\
& =\frac{1}{2 G} \int d x \delta e \wedge \delta(2 X \cosh (\alpha-\beta))+\delta \alpha \wedge \delta(2 X e \sinh (\alpha-\beta))+\delta \omega_{x} \wedge \delta \phi \\
& =\frac{1}{2 G} \int d x \delta e \wedge \delta Q^{e}+\delta \alpha \wedge \delta Q^{\alpha}+\delta \omega_{x} \wedge \delta \phi
\end{aligned}
$$

with

$$
Q^{e}:=2 X \cosh (\alpha-\beta) \quad, \quad Q^{\alpha}:=2 X e \sinh (\alpha-\beta),
$$

which provides the new canonically conjugate pairs

$$
\left\{Q^{e}(x), e(y)\right\}=\left\{Q^{\alpha}(x), \alpha(y)\right\}=\left\{\phi(x), \omega_{x}(y)\right\}=2 G \delta(x, y) .
$$

We can invert this transformation to find

$$
X^{\mp}=\frac{e Q^{e} \pm Q^{\alpha}}{2 e} \exp (\mp \alpha)
$$

and insert it into the PSM constraints:

$$
\begin{gathered}
\tilde{C}^{\mp}=\frac{1}{2 G}\left[\left(\frac{e Q^{e} \pm Q^{\alpha}}{2 e}\right)^{\prime} \mp\left(\frac{e Q^{e} \pm Q^{\alpha}}{2 e}\right)\left(\omega_{x}+\alpha^{\prime}\right) \mp \frac{1}{2} V(\phi) e\right] \exp (\mp \alpha) \\
\tilde{C}^{3}=\frac{1}{2 G}\left(\phi^{\prime}+Q^{\alpha}\right) .
\end{gathered}
$$

By the same combination as before, the diffeomorphism constraint is

$$
\tilde{D}=\frac{1}{2 G}\left(e Q^{e \prime}-Q^{\alpha} \alpha^{\prime}+\omega_{x} \phi^{\prime}\right) .
$$

\subsection{Spherically Symmetric Ashtekar Variables}

Models of loop quantum gravity are also formulated in terms of vielbein and connection components, but in different ways. The symplectic coordinate chart in this case consists of the $\mathfrak{s u}(2)$-valued Ashtekar connection $A_{a}^{i}(\bar{x})$ and the densitized triad $E_{i}^{a}(\bar{x})$ (which can be seen as an $\mathfrak{s u}(2)$-valued vector field) on a 3 d spatial manifold coordinatized by $\bar{x}$. Their 
relation to the standard canonical ADM variables is determined as follows: The spatial 3d metric $q_{a b}$ is constructed from the densitized triad via $(\operatorname{det} q) q^{a b}=E_{i}^{a} E_{i}^{b}$, and the AshtekarBarbero connection [22, 23] is related to the extrinsic curvature $K_{a}^{i}:=(\operatorname{det} E)^{-\frac{1}{2}} K_{a b} E^{b i}$ and the spin connection $\Gamma_{a}^{i}$ compatible with the triad by the formula

$$
A_{a}^{i}=\Gamma_{a}^{i}+\gamma K_{a}^{i}
$$

The real constant $\gamma>0$, called the Barbero-Immirzi parameter [23, 24], does not play a role classically because it can be changed by canonical transformations. It will become important after quantization where transformations which could change $\gamma$ are not represented unitarily.

The midisuperspace formulation in terms of these variables was initiated in [25, 26, 10, 27]. There are different spherical symmetry types on $\mathrm{SU}(2)$-principal fiber bundles, only one of which provides non-degenerate triads in its associated vector bundle [26]. Using spatial coordinates $(x, \vartheta, \varphi)$ gives the reduced densitized triad

$$
E=E^{x}(x) \tau_{3} \sin \vartheta \partial_{x}+\left(E^{1}(x) \tau_{1}+E^{2}(x) \tau_{2}\right) \sin \vartheta \partial_{\vartheta}+\left(E^{1}(x) \tau_{2}-E^{2}(x) \tau_{1}\right) \partial_{\varphi}
$$

in this non-degenerate sector, which contains only three functions $E^{x}, E^{1}$ and $E^{2}$. Here, we write $E$ as a Lie-algebra valued field using $\tau_{i}$ as a basis for $\mathfrak{s u}(2)$. The form of connections $A$ preserving the corresponding symmetry up to gauge is

$$
A=A_{x}(x) \tau_{3} d x+\left(a_{1}(x) \tau_{1}+a_{2}(x) \tau_{2}\right) d \vartheta+\left(a_{1}(x) \tau_{2}-a_{2}(x) \tau_{1}\right) \sin \vartheta d \varphi+\tau_{3} \cos \vartheta d \varphi
$$

and similarly the extrinsic curvature $K$ takes the form

$$
K=K_{x}(x) \tau_{3} d x+\left(K_{1}(x) \tau_{1}+K_{2}(x) \tau_{2}\right) d \vartheta+\left(K_{1}(x) \tau_{2}-K_{2}(x) \tau_{1}\right) \sin \vartheta d \varphi
$$

The $\mathrm{SU}(2)$-gauge freedom of the original variables leaves a residual $\mathrm{U}(1)$-gauge in the reduced theory. As before, we introduce quantities invariant under this Abelian gauge group: $A_{x}, E^{x}, K_{x}$ as they already appear in the fields together with

$$
A_{\varphi}:=\sqrt{a_{1}^{2}+a_{2}^{2}} \quad, \quad E^{\varphi}:=\sqrt{\left(E^{1}\right)^{2}+\left(E^{2}\right)^{2}} \quad, \quad K_{\varphi}:=\sqrt{K_{1}^{2}+K_{2}^{2}} .
$$

The remaining freedom of the four functions $E^{1}, E^{2}$ and $a_{1}, a_{2}$ not contained in $E^{\varphi}$ and $A_{\varphi}$ is pure gauge and can be parameterized by gauge angles $\eta(x)$ and $\tilde{\beta}(x)$, defined such that

$$
\tau_{1} \cos \eta+\tau_{2} \sin \eta:=\left(E^{1} \tau_{2}-E^{2} \tau_{1}\right) / E^{\varphi} \quad, \quad \tau_{1} \cos \tilde{\beta}+\tau_{2} \sin \tilde{\beta}:=\left(a_{1} \tau_{2}-a_{2} \tau_{1}\right) / A_{\varphi} .
$$

Each of these can be changed by the same amount with a gauge transformation, so only the difference $\tilde{\alpha}:=\eta-\tilde{\beta}$ is gauge invariant.

Relation (26) then gives [27]

$$
A_{\varphi} \cos \tilde{\alpha}=\gamma K_{\varphi} \quad, \quad A_{x}+\eta^{\prime}=\gamma K_{x} \quad \text { and } \quad A_{\varphi}^{2}=\Gamma_{\varphi}^{2}+\gamma^{2} K_{\varphi}^{2}
$$


with

$$
\Gamma_{\varphi}=-\frac{E^{x \prime}}{2 E^{\varphi}}
$$

The symplectic structure of the original variables

$$
\left\{A_{a}^{i}(\bar{x}), E_{j}^{b}(\bar{y})\right\}=8 \pi G \gamma \delta_{a}^{b} \delta_{j}^{i} \delta(\bar{x}, \bar{y})
$$

makes the functions $E^{x}, E^{1}$ and $E^{2}$ canonically conjugate to $A_{x}, a_{1}$ and $a_{2}$, respectively. However, $E^{\varphi}$ is not canonically conjugate to $A_{\varphi}$ due to the non-linear transformation from the original field components. It turns out that $E^{\varphi}$ is instead canonically conjugate to $K_{\varphi}$ [27]. The most suitable polarization for a loop quatization of spherically reduced gravity turns out to be given by the canonical pairs:

$$
\left\{A_{x}(x), \frac{1}{2 \gamma} E^{x}(y)\right\}=\left\{K_{\varphi}(x), E^{\varphi}(y)\right\}=\left\{\eta(x), \frac{1}{2 \gamma} P^{\eta}(y)\right\}=G \delta(x, y) .
$$

The reduced $2 \mathrm{~d}$ action is then

$$
S=\int d t\left[\frac{1}{2 G \gamma} \int d x\left(E^{x} \dot{A}_{x}+2 \gamma E^{\varphi} \dot{K}_{\varphi}+P^{\eta} \dot{\eta}\right)-\int d x\left(\lambda \tilde{\mathcal{G}}_{\text {grav }}+N^{x} \tilde{\mathcal{D}}_{\text {grav }}+N \tilde{\mathcal{H}}_{\text {grav }}\right)\right]
$$

with the Gauss constraint

$$
G_{\text {grav }}[\lambda]=\frac{1}{2 G \gamma} \int d x \lambda\left(E^{x \prime}+P^{\eta}\right)
$$

generating $\mathrm{U}(1)$-gauge transformations, the diffeomorphism constraint

$$
D_{\text {grav }}\left[N^{x}\right]=\frac{1}{2 G} \int d x N^{x}\left(2 E^{\varphi} K_{\varphi}^{\prime}-K_{x} E^{x \prime}+\frac{1}{\gamma} \eta^{\prime}\left(E^{x \prime}+P^{\eta}\right)\right)
$$

generating diffeomorphisms on the one dimensional radial manifold and the Hamiltonian constraint

$$
H_{\text {grav }}[N]=-\frac{1}{2 G} \int d x N\left|E^{x}\right|^{-\frac{1}{2}}\left(K_{\varphi}^{2} E^{\varphi}+2 K_{\varphi} K_{x} E^{x}+\left(1-\Gamma_{\varphi}^{2}\right) E^{\varphi}+2 \Gamma_{\varphi}^{\prime} E^{x}\right)
$$

generating dynamical evolution.

\section{Relating the two models}

The actions (16) and (32) represent equivalent canonical formulations of spherically reduced general relativity, so there must exist a canonical transformation between the PSM and Ashtekar variables. To find such a transformation we first compare the form of the reduced 2 -dimensional metric in terms of these two sets of variables. This relates the dilaton field $\phi$ and the gauge invariant part $e$ of the dyad directly to the densitized triad components $E^{x}$ and $E^{\varphi}$. Using this and imposing the canonical relations (21) gives a system of differential equations for the remaining PSM variables. We then use equations of motion (8) to fix some of the ambiguities and check for consistency. 


\subsection{Comparison of metrics}

The general canonical line element $d s^{2}=-N^{2} d t^{2}+q_{a b}\left(d x^{a}+N^{a} d t\right)\left(d x^{b}+N^{b} d t\right)$ adapted to spherical symmetry with coordinates $(t, x, \vartheta, \varphi)$, lapse function $N(t, x)$ and shift vector $N^{x}(t, x)$ is

$$
d s^{2}=-N(t, x)^{2} d t^{2}+q_{x x}(t, x)\left(d x+N^{x}(t, x) d t\right)^{2}+q_{\varphi \varphi}(t, x) d \Omega^{2}
$$

where $q_{x x}$ and $q_{\varphi \varphi}$ are components of the spatial metric $d q^{2}=q_{x x}(t, x) d x^{2}+q_{\varphi \varphi}(t, x) d \Omega^{2}$. In terms of the densitized triad, we have

$$
q_{x x}=\frac{E^{\varphi 2}}{\left|E^{x}\right|} \quad, \quad q_{\varphi \varphi}=\left|E^{x}\right| .
$$

Comparing this form of the metric with (11) directly gives

$$
q_{\varphi \varphi}=\Phi^{2} \quad, \quad \mathbf{g}_{\mu \nu}=\left(\begin{array}{cc}
-N^{2}+q_{x x}\left(N^{x}\right)^{2} & q_{x x} N^{x} \\
q_{x x} N^{x} & q_{x x}
\end{array}\right)
$$

This relates the densitized spherically symmetric triad variables to the dyads of the conformally transformed metric $g_{\mu \nu}=\sqrt{\phi} \mathbf{g}_{\mu \nu}$ and the dilaton field $\phi$ :

$$
\begin{aligned}
\phi=\Phi^{2} & =\left|E^{x}\right| \\
g=2 e^{+} e^{-} & =\sqrt{\phi} \mathbf{g} \\
\left(\begin{array}{ccc}
2 e_{t}^{+} e_{t}^{-} & e_{t}^{+} e_{x}^{-}+e_{x}^{+} e_{t}^{-} \\
e_{t}^{+} e_{x}^{-}+e_{x}^{+} e_{t}^{-} & 2 e_{x}^{+} e_{x}^{-}
\end{array}\right) & =\left|E^{x}\right|^{\frac{1}{2}}\left(\begin{array}{cc}
-N^{2}+\frac{E^{\varphi 2}}{\mid E^{x \mid}} N^{x 2} & \frac{E^{\varphi 2}}{\left|E^{x}\right|} N^{x} \\
\frac{E^{\varphi} 2^{x}}{\left|E^{x}\right|} N^{x} & \frac{E^{\varphi}}{\left|E^{x}\right|}
\end{array}\right) .
\end{aligned}
$$

From this we obtain

$$
e^{2}=e_{x}^{+} e_{x}^{-}=\frac{E^{\varphi 2}}{2\left|E^{x}\right|^{\frac{1}{2}}}
$$

and

$$
\begin{aligned}
e_{x}^{+}=p \frac{E^{\varphi}}{\sqrt{2}\left|E^{x}\right|^{\frac{1}{4}}} \exp \alpha \quad, \quad e_{x}^{-}=p \frac{E^{\varphi}}{\sqrt{2}\left|E^{x}\right|^{\frac{1}{4}}} \exp (-\alpha) \\
e_{t}^{+}=p \frac{N^{x} E^{\varphi} \pm N\left|E^{x}\right|^{\frac{1}{2}}}{\sqrt{2}\left|E^{x}\right|^{\frac{1}{4}}} \exp \alpha \quad, \quad e_{t}^{-}=p \frac{1}{\sqrt{2}\left|E^{x}\right|^{\frac{1}{4}}}\left(\frac{-N^{2}\left|E^{x}\right|+N^{x} E^{\varphi} E^{2}}{N^{x} E^{\varphi} \pm N\left|E^{x}\right|^{\frac{1}{2}}}\right) \exp (-\alpha)
\end{aligned}
$$

with $p= \pm 1$ distinguishing different solutions.

Using equations (8) and (10) and the equations of motion

$$
\begin{aligned}
& \dot{E}^{x}=2 s N K_{\varphi}\left|E^{x}\right|^{\frac{1}{2}}+N^{x} E^{x \prime} \\
& \dot{E}^{\varphi}=N\left(K_{\varphi} E^{\varphi}+K_{x} E^{x}\right)\left|E^{x}\right|^{-\frac{1}{2}}+\left(N^{x} E^{\varphi}\right)^{\prime}
\end{aligned}
$$


for the spherically symmetric loop variables where $s$ is the sign of $E^{x}$, we get the dependence of the spin connection $\omega_{x}$ and Lagrange multipliers $X^{ \pm}$in terms of $\left(E^{x}, E^{\varphi}, K_{x}, K_{\varphi}\right)$ :

$$
\omega_{x}= \pm s K_{x} \pm \frac{E^{\varphi}}{2 \mid E^{x \mid}} K_{\varphi}-\alpha^{\prime}
$$

and

$$
\begin{aligned}
& X^{-}=p \sqrt{2}\left|E^{x}\right|^{\frac{1}{4}}\left(-s \frac{E^{x \prime}}{2 E^{\varphi}} \mp K_{\varphi}\right) \exp (-\alpha) \\
& X^{+}=p \sqrt{2}\left|E^{x}\right|^{\frac{1}{4}}\left(s \frac{E^{x \prime}}{2 E^{\varphi}} \mp K_{\varphi}\right) \exp \alpha .
\end{aligned}
$$

\subsection{Canonical transformation}

We now look for a canonical transformation between the two sets of variables

$$
\left(Q^{e}, Q^{\alpha}, \phi ; e, \alpha, \omega_{x}\right) \quad \leftrightarrows \quad\left(E^{x}, E^{\varphi}, P^{\eta} ; A_{x}, K_{\varphi}, \eta\right)
$$

The Poisson bracket relations (21) give a system of partial differential equations for the functional dependence of $Q^{e}, Q^{\alpha}, \alpha$, and $\omega_{x}$ on the spherically symmetric loop variables. (There are 15 nontrivial relations that must be simultaneously satisfied to ensure consistency: $\left\{Q^{e}, e\right\}=2 G,\left\{Q^{e}, \phi\right\}=\left\{Q^{\alpha}, e\right\}=\left\{Q^{\alpha}, \phi\right\}=0,\left\{\phi, \omega_{x}\right\}=2 G,\left\{e, \omega_{x}\right\}=\{\phi, \alpha\}=$ $\{e, \alpha\}=0,\left\{Q^{\alpha}, \alpha\right\}=2 G,\left\{Q^{e}, \alpha\right\}=\left\{Q^{e}, Q^{\alpha}\right\}=\left\{Q^{e}, \omega_{x}\right\}=\left\{Q^{\alpha}, \omega_{x}\right\}=\left\{\alpha, \omega_{x}\right\}=0$. The remaining $\{\phi, e\}=0$ is automatically satisfied given the functional dependence of $\phi$ and $e$ on $E^{x}$ and $E^{\varphi}$.)

These equations are solved in App. A, providing the canonical transformation

$$
\begin{aligned}
Q^{e}=p 2 \sqrt{2}\left|E^{x}\right|^{\frac{1}{4}} K_{\varphi}+h\left[\left|E^{x}\right|^{-\frac{1}{4}} E^{\varphi}\right] \quad, \quad e=p \frac{E^{\varphi}}{\sqrt{2}\left|E^{x}\right|^{\frac{1}{4}}} \\
\phi=\left|E^{x}\right| \quad, \quad \omega_{x}=-s K_{x}-\frac{E^{\varphi}}{2\left|E^{x}\right|} K_{\varphi}+\frac{1}{k} \eta^{\prime} \\
Q^{\alpha}=\frac{k}{\gamma} P^{\eta}+\left(\frac{k-s \gamma}{\gamma}\right) E^{x \prime} \quad, \quad \alpha=-\frac{1}{k} \eta
\end{aligned}
$$

with inverse transformation

$$
\begin{array}{rlrl}
E^{x}=s \phi & , & E^{\varphi}=p \sqrt{2} \phi^{\frac{1}{4}} e \\
K_{\varphi}=p \frac{\left(Q^{e}-h\right)}{2 \sqrt{2} \phi^{\frac{1}{4}}} & , & K_{x} & =-s\left(\omega_{x}+\alpha^{\prime}+\frac{e}{4 \phi}\left(Q^{e}-h\right)\right) \\
\eta=-k \alpha & , & P^{\eta}=\frac{\gamma}{k} Q^{\alpha}+\left(\frac{\gamma-s k}{k}\right) \phi^{\prime} .
\end{array}
$$

Here again, $s=\operatorname{sign}\left(E^{x}\right), k$ is an arbitrary constant, and $h$ an arbitrary function of one variable. 


\subsection{Constraints}

We take $h=0$ to provide a specific canonical transformation. As mentioned in App. A, with this solution the $C^{3}$ constraint (24) reproduces the Gauss constraint (33) : 2

$$
C^{3}[\lambda]=\frac{1}{2 G} \int d x \lambda\left(\phi^{\prime}+Q^{\alpha}\right)=\frac{k}{2 G \gamma} \int d x \lambda\left(E^{x \prime}+P^{\eta}\right)=k G_{\text {grav }}[\lambda] .
$$

The diffeomorphism constraint (25) reads:

$$
\begin{aligned}
D\left[N^{x}\right] & =\frac{1}{2 G} \int d x N^{x}\left(e Q^{e \prime}-Q^{\alpha} \alpha^{\prime}+\omega_{x} \phi^{\prime}\right) \\
& =\frac{1}{2 G} \int d x N^{x}\left(2 E^{\varphi} K_{\varphi}^{\prime}-K_{x} E^{x \prime}+\gamma^{-1} \eta^{\prime}\left(P^{\eta}+E^{x \prime}\right)\right)=D_{\text {grav }}\left[N^{x}\right]
\end{aligned}
$$

Using (29), the remaining independent linear combination becomes

$$
\begin{aligned}
& C^{+}\left[\frac{\sqrt{2}}{2} N \phi^{1 / 4} \exp (-\alpha)\right]-C^{-}\left[\frac{\sqrt{2}}{2} N \phi^{1 / 4} \exp (\alpha)\right]=\frac{\sqrt{2}}{4 G} \int d x N \phi^{1 / 4}\left[Q^{e}\left(\omega_{x}+\alpha^{\prime}\right)-\left(\frac{Q^{\alpha}}{e}\right)^{\prime}+V e\right] \\
&=\frac{p}{2 G} \int d x N[-\left|E^{x}\right|^{-\frac{1}{2}} K_{\varphi}^{2} E^{\varphi}-2 s\left|E^{x}\right|^{\frac{1}{2}} K_{x} K_{\varphi}+\frac{E^{\varphi} V}{2} \\
&\left.+\frac{\left|E^{x}\right|^{-\frac{1}{2}} E^{x / 2}}{4 E^{\varphi}}-\frac{s\left|E^{x}\right|^{\frac{1}{2}} E^{x \prime} E^{\varphi \prime}}{E^{\varphi} 2}+\frac{s\left|E^{x}\right|^{\frac{1}{2}} E^{x \prime \prime}}{E^{\varphi}}\right] \\
&-\frac{p k}{2 G \gamma} \int d x N\left|E^{x}\right|^{1 / 4}\left[\frac{\left|E^{x}\right|^{\frac{1}{4}}}{E^{\varphi}}\left(E^{x \prime}+P^{\eta}\right)\right]^{\prime}
\end{aligned}
$$

and reproduces the Hamiltonian constraint (35) with $V(\phi)=-\frac{2}{\sqrt{\phi}}=-2\left|E^{x}\right|^{-\frac{1}{2}}$ (up to the Gauss constraint):

$C^{+}\left[\frac{\sqrt{2}}{2} N \phi^{1 / 4} \exp (-\alpha)\right]-C^{-}\left[\frac{\sqrt{2}}{2} N \phi^{1 / 4} \exp (\alpha)\right]=p H_{\text {grav }}[N]-\frac{p k}{2 G \gamma} \int d x N\left|E^{x}\right|^{1 / 4}\left[\frac{\left|E^{x}\right| \frac{1}{4}}{E^{\varphi}}\left(E^{x^{\prime}}+P^{\eta}\right)\right]^{\prime}$.

To summarize,

$$
\begin{aligned}
\tilde{C}^{3} & =k \tilde{\mathcal{G}}_{\text {grav }} \\
e \exp (\alpha) \tilde{C}^{-}+e \exp (-\alpha) \tilde{C}^{+}+\omega_{x} \tilde{C}^{3} & =\tilde{\mathcal{D}}_{\text {grav }} \\
-\exp (\alpha) \tilde{C}^{-}+\exp (-\alpha) \tilde{C}^{+}+\left(\frac{\tilde{C}^{3}}{e}\right)^{\prime} & =\sqrt{2} p \phi^{-\frac{1}{4}} \tilde{\mathcal{H}}_{\text {grav }}
\end{aligned}
$$

verifying once more that (16) and (32) represent equivalent constrained systems.

\footnotetext{
${ }^{2}$ There is a local agreement of the infinitesimal Gauss symmetries generated by the constraints. Globally, however, the formulations differ, one having a compact group $\mathrm{U}(1)$, the other the noncompact $\mathrm{SO}(1,1)$. In fact, different time gauges have been used to reduce space-time metrics to objects in canonical form, which turns out to imply different topological properties of the gauge orbits.
} 


\section{Inclusion of Yang-Mills fields}

Before discussing our main topic, the role of quantum corrections, we extend the formalism to include 2-dimensional Yang-Mills fields [11]. This will provide a non-trivial model in the presence of quantum corrections. The general 2-dimensional Yang-Mills action with an arbitrary coupling $\zeta$, allowed to depend on the dilaton field $\phi$, reads

$$
S_{\mathrm{YM}}=-\frac{1}{4} \int_{M} \zeta \operatorname{tr}(\mathcal{F} \wedge * \mathcal{F})
$$

where $\mathcal{F}^{I}=d \mathcal{A}^{I}+\frac{1}{2} c^{I}{ }_{J K} \mathcal{A}^{J} \wedge \mathcal{A}^{K}$ is the usual curvature 2 -form of the connection $\mathcal{A}^{I} T_{I}$, with $T_{I}$ a basis for the Lie algebra $\mathfrak{g}$ with structure constants $c^{I}{ }_{J K}$ of the chosen $n$-dimensional internal gauge group. (It should be stressed that from a physical point of view this is a toy model; spherically reduced Yang-Mills theory contains extra fields and does not coincide with the purely $2 \mathrm{~d}$ model.)

In first order form this action is

$$
S_{\mathrm{YM}}=-\int_{M} \mathcal{E}^{I} \mathcal{F}_{I}+2 \zeta(\phi) \mathcal{E}^{I} \mathcal{E}_{I} \varepsilon
$$

(Assuming $\operatorname{tr}\left(T_{I} T_{J}\right)=\frac{1}{2} \delta_{I J}$, this equivalence is seen most easily by inserting the field equation $\mathcal{E}^{I}=\frac{1}{4 \zeta} * \mathcal{F}^{I}$ into the original Yang-Mills action.) Then $S_{\text {grav }}+S_{\text {YM }}$ reads

$$
S_{\text {gravYM }}=-\frac{1}{2 G} \int_{M} X^{a} D e_{a}+\phi d \omega+2 G \mathcal{E}^{I} \mathcal{F}_{I}+\left(\frac{1}{2} V(\phi)+4 G \zeta(\phi) \mathcal{E}^{I} \mathcal{E}_{I}\right) \varepsilon
$$

where indices $a$ run over + and - , and $I=1, \ldots, n$. The coupling of Yang-Mills theory to gravity thus changes the dilaton potential in a way which depends on the value of the dilaton through the coupling function $\zeta$. Moreover, the target manifold of the PSM has a higher dimension due to the degrees of freedom of the Yang-Mills field: After integrating by parts and dropping the corresponding surface terms, and with the identifications

$$
\left(\mathbf{X}^{i}\right):=\left(X^{a}, \phi, \mathcal{E}^{I}\right) \quad, \quad\left(\mathbf{A}_{i}\right):=\left(e_{a}, \omega, \mathcal{A}_{I}\right)
$$

the previous action turns out to be of Poisson sigma form (2) on an $(n+3)$-dimensional target space $N$ with Poisson brackets

$$
\begin{array}{rll}
\left\{X^{+}, X^{-}\right\}=V / 2+4 G \zeta \mathcal{E}^{I} \mathcal{E}_{I} & , & \left\{X^{ \pm}, \phi\right\}= \pm X^{ \pm} \\
\left\{\mathcal{E}^{I}, \mathcal{E}^{J}\right\}=c^{I J}{ }_{K} \mathcal{E}^{K} & , & \left\{X^{ \pm}, \mathcal{E}^{I}\right\}=\left\{\phi, \mathcal{E}^{I}\right\}=0 .
\end{array}
$$

The Poisson bivector can thus be decomposed as

$$
\left(\mathcal{P}^{i j}\right)=\left[\begin{array}{ll}
\mathbf{P} & 0 \\
0 & \mathbf{Q}
\end{array}\right]
$$


with the $3 \times 3$ matrix

$$
\mathbf{P}=\left(\begin{array}{ccc}
0 & -V / 2-4 G \zeta \mathcal{E}^{I} \mathcal{E}_{I} & -X^{-} \\
V / 2+4 G \zeta \mathcal{E}^{I} \mathcal{E}_{I} & 0 & X^{+} \\
X^{-} & -X^{+} & 0
\end{array}\right)
$$

and the $n \times n$ matrix

$$
\mathbf{Q}=\left(Q^{I J}\right)=\left(c^{I J}{ }_{K} \mathcal{E}^{K}\right) .
$$

The canonical formulation of Sec. 2.1.3 proceeds almost unchanged: The symplectic structure (30) is extended by the Yang-Mills pairs

$$
\left\{\mathcal{E}^{I}(x), \mathcal{A}_{I}(y)\right\}=\delta(x, y) .
$$

The constraints $C^{\mp}($ (23) $)$ (and (84) $)$ receive additional terms $\mp 2 \zeta \mathcal{E}^{I} \mathcal{E}_{I} e \exp (\mp \alpha)$ :

$$
\tilde{C}^{\mp}=\frac{1}{2 G}\left[\left(\frac{e Q^{e} \pm Q^{\alpha}}{2 e}\right)^{\prime} \mp\left(\frac{e Q^{e} \pm Q^{\alpha}}{2 e}\right)\left(\omega_{x}+\alpha^{\prime}\right) \mp \frac{1}{2} V(\phi) e \mp 4 G \zeta \mathcal{E}^{I} \mathcal{E}_{I} e\right] \exp (\mp \alpha)
$$

and we have the usual $n$-component Gauss constraints

$$
\tilde{C}^{3+I}=\mathcal{E}^{I \prime}+c_{K}^{I J} \mathcal{A}_{J} \mathcal{E}^{K}
$$

for the Yang-Mills part of the theory.

\section{Loop quantization of general 2-dimensional dilaton gravity}

We can now obtain the first application of our canonical relation between dilaton gravity and spherically symmetric gravity in Ashtekar variables: a loop quantization of general 2-dimensional dilaton gravity models. So far in this context, loop quantizations have only been performed for one specific dilaton potential $V(\phi) \propto 1 / \sqrt{\phi}$, corresponding to spherically symmetric gravity in four dimensions [10]. Looking at the Hamiltonian constraint in the form (46) shows that the potential appears only at one place, which is in fact a rather simple term in the Hamiltonian (resulting from the term $E^{\varphi}$ in the parenthesis in (35), which is the only term independent of $K_{\varphi}$ or $\Gamma_{\varphi}$ ). This observation allows us immediately to extend the existing loop quantization of spherically symmetric gravity to an arbitrary potential $V(\phi)$, provided only that the expression $V(\phi)$ can be turned into a well-defined operator. Since this is merely a function of the triad component $E^{x}$, such quantizations easily exist.

\subsection{Loop quantization}

To quantize spherically symmetric gravity, we have to define a well-defined algebra of quantities which seperate points on the classical phase space of the fields $\left(A_{x}, K_{\varphi}, \eta ; E^{x}, E^{\varphi}, P^{\eta}\right)$. 
In particular, some of the fields must be integrated ("smeared") in suitable ways so as to provide an algebra under taking Poisson brackets free of the delta functions which appear for the fields in (31). The resulting algebra is then well-defined and can be represented on a Hilbert space to provide the basic representation of the quantum theory. In gravitational systems, the smearing must be done with care because there is no background metric to define the integrations (in addition to the physical metric given by $E^{x}$ and $E^{\varphi}$ which are to be quantized).

A loop quantization is based on holonomies

$$
h_{e}\left[A_{x}\right]=\exp \left(\frac{1}{2} i \int_{e} A_{x} d x\right) \quad, \quad h_{v}\left[K_{\varphi}\right]=\exp \left(i \gamma K_{\varphi}(v)\right) \quad, \quad h_{v}[\eta]=\exp (i \eta(v))
$$

as smeared versions of the configuration variables. Here, we have used arbitrary curves $e$ and points $v$ in the radial line as labels. Varying $e$ and $v$ allows one to recover smooth local fields unambiguously. The appearance of integrations without auxiliary structures is dictated by the tensorial nature of the variables: The $\mathrm{U}(1)$-connection $A_{x}$ can naturally be integrated to define parallel transport, while the remaining components are scalars which we simply exponentiate without integrations. In this framework, exponentiations are not strictly necessary, but we use them in order to take into account the origin of these objects from non-Abelian holonomies in the full setting. Using exponentials instead of linear expressions in connection or extrinsic curvature components will not spoil the linear nature of the underlying basic algebra; see Eq. (56) below.

Similarly, we define flux variables

$$
F_{v}\left[E^{x}\right]=E^{x}(v) \quad, \quad F_{e}\left[E^{\varphi}\right]=\int_{e} E^{\varphi} d x \quad, \quad F_{e}\left[P^{\eta}\right]=\int_{e} P^{\eta} d x
$$

for the momenta. Also here, the integrations are naturally dictated by transformation properties of the fields, $E^{x}$ being scalar while $E^{\varphi}$ and $P^{\eta}$ are densities of weight one. Without introducing a background metric or integration measure, we have thus managed to integrate all fields such that a well-defined algebra results:

$$
\begin{aligned}
\left\{h_{e}\left[A_{x}\right], F_{v}\left[E^{x}\right]\right\} & =i \gamma G \delta_{v \in e} h_{e}\left[A_{x}\right] \\
\left\{h_{v}\left[K_{\varphi}\right], F_{e}\left[E^{\varphi}\right]\right\} & =i \gamma G \delta_{v \in e} h_{v}\left[K_{x}\right] \\
\left\{h_{v}[\eta], F_{e}\left[P^{\eta}\right]\right\} & =2 i \gamma G \delta_{v \in e} h_{v}[\eta]
\end{aligned}
$$

where $\delta_{v \in e}$ is one if $v \in e$ and zero otherwise.

An irreducible representation of this algebra can easily be constructed. In the connection representation (which is customarily used in the full theory), an orthonormal basis of states is given by

$$
T_{g, k, \mu}\left[A_{x}, K_{\varphi}, \eta\right]=\prod_{e \in g} \exp \left(\frac{1}{2} i k_{e} \int_{e} A_{x} d x\right) \prod_{v \in g} \exp \left(i \mu_{v} \gamma K_{\varphi}(v)\right) \exp \left(i k_{v} \eta(v)\right)
$$


with integer labels $k_{e}, k_{v}$ and positive real labels $\mu_{v}$ on edges $e$ and vertices $v$, respectively, forming a finite graph $g$ in the 1-dimensional radial line. The labels determine the connection dependence by irreducible representations of the groups spanned by the holonomies. (These groups are $\mathrm{U}(1)$ for $A_{x^{-}}$and $\eta$-holonomies and the Bohr compactification $\overline{\mathbb{R}}_{\text {Bohr }}$ of the real line for $K_{\varphi}$-holonomies; see [10] for details.)

Holonomies then simply act as multiplication operators. Specifically:

$$
\hat{h}_{e}\left[A_{x}\right] T_{g, k, \mu}=T_{g, k+\delta_{e}, \mu} \quad, \quad \hat{h}_{v}\left[K_{\varphi}\right] T_{g, k, \mu}=T_{g, k, \mu+\delta_{v}} \quad, \quad \hat{h}_{v}[\eta] T_{g, k, \mu}=T_{g, k+\delta_{v}, \mu}
$$

where $\delta_{e}$ is a function on the set of all edges which is one for the edge $e$ and zero otherwise, and analogously for the function $\delta_{v}$ on the set of vertices. The state $T_{g, 0,0}\left[A_{x}, K_{\varphi}, \eta\right]=1$ is cyclic for this representation.

The densitized triad components, which are momenta conjugate to the connection components, act as derivative operators:

$$
\begin{aligned}
\hat{F}_{v}\left[E^{x}\right] T_{g, k, \mu} & =\gamma \ell_{\mathrm{P}}^{2} \frac{k_{e^{+}(v)}+k_{e^{-}(v)}}{2} T_{g, k, \mu} \\
\hat{F}_{e}\left[E^{\varphi}\right] T_{g, k, \mu} & =\gamma \ell_{\mathrm{P}}^{2} \sum_{v \in e} \mu_{v} T_{g, k, \mu} \\
\hat{F}_{e}\left[P^{\eta}\right] T_{g, k, \mu} & =2 \gamma \ell_{\mathrm{P}}^{2} \sum_{v \in e} k_{v} T_{g, k, \mu}
\end{aligned}
$$

where $\ell_{\mathrm{P}}^{2}=G \hbar$ is the Planck length squared and $e^{ \pm}(v)$ denote the edges neighboring a point $v$, distinguished from each other using a given orientation of the radial line. (We have $k_{e^{+}(v)}=k_{e^{-}(v)}$ if $v$ is not a vertex of the graph.) All flux operators have discrete spectra: eigenstates as seen in (59) and (60) are normalizable. But only $\hat{E}^{x}$ has a discrete set of eigenvalues, while $\hat{E}^{\varphi}$-eigenvalues fill the real line. (Their eigenstates are elements of the non-separable Hilbert space of square integrable functions on the Bohr compactification of the real line.)

These basic operators can be used for composite operators as well, providing welldefined but rather complicated constraint operators. The Gauss constraint is linear in triad components and can directly be quantized in terms of the basic flux operators and implies

$$
k_{v}=-\frac{1}{2}\left(k_{e^{+}(v)}-k_{e^{-}(v)}\right) .
$$

A basis for gauge invariant states in the kernel of the Gauss constraint is thus

$$
\bar{T}_{g, k, \mu}\left[A_{x}+\eta^{\prime}, K_{\varphi}\right]=\prod_{e \in g} \exp \left(\frac{1}{2} i k_{e} \int_{e}\left(A_{x}+\eta^{\prime}\right) d x\right) \prod_{v \in g} \exp \left(i \mu_{v} \gamma K_{\varphi}(v)\right)
$$

where the labels $k_{v}$ are eliminated by imposing (62). Accordingly, states solving the Gauss constraint only depend on $A_{x}+\eta^{\prime}$, not on $A_{x}$ and $\eta^{\prime}$ separately. The diffeomorphism constraint can directly be represented in its finite version, where its action simply moves labelled graphs in the radial manifold by a spatial diffeomorphism $\Phi: \Phi T_{g, k, \mu}=T_{\Phi(g, k, \mu)}$ where $\Phi(g, k, \mu)=\left(\Phi(g), k^{\prime}, \mu^{\prime}\right)$ is the graph $\Phi(g)$ with labels $k_{\Phi(e)}^{\prime}=k_{e}$ and $\mu_{\Phi(v)}^{\prime}=\mu_{v}$. 
Operators quantizing the Hamiltonian constraint have been constructed, but their constraint equations for physical states are more difficult to solve. Several steps are involved in this particular case: First, we have to quantize the inverse of $E^{x}$ as it appears in the classical constraint. There is no direct operator inverse because $\hat{E}^{x}$ has a discrete spectrum containing zero. Nevertheless, well-defined operators with the correct classical limit exist, which are based on quantizing, e.g., the right hand side of the classical identity

$$
4 \pi \gamma G \frac{\operatorname{sgn}\left(E^{x}\right) E^{\varphi}}{\sqrt{\mid E^{x \mid}}}=\left\{A_{x}, V\right\}=2 i h_{e}\left[A_{x}\right]\left\{h_{e}\left[A_{x}\right]^{-1}, V\right\}
$$

instead of the left hand side [4]. This can be done using a quantization of the spatial volume $V=4 \pi \int d x E^{\varphi} \sqrt{\mid E^{x \mid}}$ in terms of fluxes and turning the Poisson bracket into a commutator divided by $i \hbar$. Secondly, connection and extrinsic curvature components in the classical constraint are turned into holonomies and then quantized directly using the basic operators. Finally, one can quantize the spin connection terms making use of discretizations of the spatial derivatives. In this process, a well-defined operator

$$
\begin{aligned}
\hat{H}[N]= & \frac{i}{2 \pi G \gamma^{3} \delta^{2} \ell_{\mathrm{P}}^{2}} \sum_{v, \sigma= \pm 1} \sigma N(v) \operatorname{tr}\left(\left(h_{\vartheta} h_{\varphi} h_{\vartheta}^{-1} h_{\varphi}^{-1}-h_{\varphi} h_{\vartheta} h_{\varphi}^{-1} h_{\vartheta}^{-1}\right.\right. \\
& \left.+2 \gamma^{2} \delta^{2}\left(1-\hat{\Gamma}_{\varphi}^{2}\right) \tau_{3}\right) h_{x, \sigma}\left[h_{x, \sigma}^{-1}, \hat{V}\right] \\
& +\left(h_{x, \sigma} h_{\vartheta}\left(v+e^{\sigma}(v)\right) h_{x, \sigma}^{-1} h_{\vartheta}(v)^{-1}-h_{\vartheta}(v) h_{x, \sigma} h_{\vartheta}\left(v+e^{\sigma}(v)\right)^{-1} h_{x, \sigma}^{-1}\right. \\
& \left.+2 \gamma^{2} \delta \int_{e^{\sigma}(v)} \hat{\Gamma}_{\varphi}^{\prime} \Lambda(v)\right) h_{\varphi}\left[h_{\varphi}^{-1}, \hat{V}\right] \\
& +\left(h_{\varphi}(v) h_{x, \sigma} h_{\varphi}\left(v+e^{\sigma}(v)\right)^{-1} h_{x, \sigma}^{-1}-h_{x, \sigma} h_{\varphi}\left(v+e^{\sigma}(v)\right) h_{x, \sigma}^{-1} h_{\varphi}(v)^{-1}\right. \\
& \left.\left.+2 \gamma^{2} \delta \int_{e^{\sigma}(v)} \hat{\Gamma}_{\varphi}^{\prime} \bar{\Lambda}(v)\right) h_{\vartheta}\left[h_{\vartheta}^{-1}, \hat{V}\right]\right)
\end{aligned}
$$

with the correct classical limit results. Here, $\delta$ is a parameter appearing in the exponent of angular holonomies $h_{\vartheta}=\exp \left(\delta K_{\varphi} \tau_{1}\right)$ and $h_{\varphi}=\exp \left(\delta K_{\varphi} \tau_{2}\right)$. We use the $\mathrm{SU}(2)$-form of holonomies for compactness and for an easier comparison with the full theory; as matrix elements we have our basic holonomies $h_{v}\left[K_{\varphi}\right]$ from $h_{\vartheta}$ and $h_{\varphi}$, as well as $h_{e^{\sigma}}(v)\left[A_{x}\right]$ from $h_{x, \sigma}(v):=\exp \left(\int_{e^{\sigma}(v)} A_{x} \tau_{3} d x\right)$. Moreover, matrix elements of $\Lambda(v):=\tau_{1} \cos \eta(v)+\tau_{2} \sin \eta(v)$ and $\bar{\Lambda}(v):=-\tau_{1} \sin \eta(v)+\tau_{2} \cos \eta(v)$ act by multiplication with holonomies $h_{v}[\eta]$. The parameter $\delta$ in angular holonomies may be a function of $E^{x}$ instead of a constant; this represents the possibility of lattice refinements [28, 29] in the symmetry orbits which a full Hamiltonian constraint operator in general implies since it creates new edges in a graph by acting with the corresponding holonomies. In the reduced model, there are no edges in the symmetry orbits, and thus no direct way exists to implement the creation of such new edges. Instead, an $E^{x}$-dependence of $\delta$ can be used to make the edge parameter $\delta$ depend on the area of the orbit. A value $\delta$ shrinking with the area would imply that more edges 
are created because the coordinate length of each new edge used is smaller at a larger areal size.

The constraint equation $\hat{H}[N] \psi=0$ for all $N$ can be formulated as a set of coupled difference equations for states labeled by the triad quantum numbers $k_{e}$ and $\mu_{v}$, which have the form

$$
\begin{aligned}
& \hat{C}_{\mathrm{R}+}\left(k_{-}, k_{+}-2\right)^{\dagger} \psi\left(\ldots, k_{-}, k_{+}-2, \ldots\right)+\hat{C}_{\mathrm{R}-}\left(k_{-}, k_{+}+2\right)^{\dagger} \psi\left(\ldots, k_{-}, k_{+}+2, \ldots\right) \\
+ & \hat{C}_{\mathrm{L}+}\left(k_{-}-2, k_{+}\right)^{\dagger} \psi\left(\ldots, k_{-}-2, k_{+}, \ldots\right)+\hat{C}_{\mathrm{L}-}\left(k_{-}+2, k_{+}\right)^{\dagger} \psi\left(\ldots, k_{-}+2, k_{+}, \ldots\right) \\
+ & \hat{C}_{0}\left(k_{-}, k_{+}\right)^{\dagger} \psi\left(\ldots, k_{-}, k_{+}, \ldots\right)=0
\end{aligned}
$$

one for each vertex. Only the edge labels $k_{e}$ are written explicitly in this difference expression, but states also depend on vertex labels $\mu_{v}$ on which the coefficient operators $\hat{C}_{I}$ act; see [27] for details. We will only require the central coefficient

$$
\begin{aligned}
\hat{C}_{0}|\vec{\mu}, \vec{k}\rangle= & \frac{\ell_{\mathrm{P}}}{2 \sqrt{2} G \gamma^{3 / 2} \delta^{2}}\left(|\mu|\left(\sqrt{\left|k_{+}+k_{-}+1\right|}-\sqrt{\left|k_{+}+k_{-}-1\right|}\right)\right. \\
\times & \left(\left|\mu_{-}, k_{-}, \mu+2 \delta, k_{+}, \mu_{+}\right\rangle+\left|\mu_{-}, k_{-}, \mu-2 \delta, k_{+}, \mu_{+}\right\rangle\right. \\
& \left.-2\left(1+2 \gamma^{2} \delta^{2}\left(1-\Gamma_{\varphi}^{2}(\vec{\mu}, \vec{k})\right)\right)\left|\mu_{-}, k_{-}, \mu, k_{+}, \mu_{+}\right\rangle\right) \\
& \left.-4 \gamma^{2} \delta^{2} \operatorname{sgn}_{\delta / 2}(\mu) \sqrt{\mid k_{+}+k_{-}} \Gamma_{\varphi}^{\prime}(\vec{\mu}, \vec{k})\left|\mu_{-}, k_{-}, \mu, k_{+}, \mu_{+}\right\rangle\right) \\
+ & \hat{H}_{\text {matter }, v}\left|\mu_{-}, k_{-}, \mu, k_{+}, \mu_{+}\right\rangle
\end{aligned}
$$

below. For these basic difference equations, one can show that they are free of gravitational singularities in the sense of quantum hyperbolicity [30]: the recurrence scheme they define provides an evolution of the wave function which does not stop where a classical singularity would form [31].

These difference equations are linear but quite involved; moreover, they are formulated for a wave function which is not easy to interpret in a generally covariant system. Instead of solving the equations directly, one can make use of an effective analysis which provides approximate equations of a simpler form for expectation values and possibly higher moments of the state. These equations are obtained as a Hamiltonian system whose Hamiltonian function is the expectation value of the Hamiltonian operator in a general state [7, and which are constrained by expectation values of products of constraint operators [32]. Through the expectation value, characteristic features of the quantized Hamiltonian, such as deviations of the quantized $1 / E^{x}$ from the classical behavior, enter the effective Hamiltonian and give rise to potential physical effects (e.g. for black hole collapse in [29]). We will later use this type of corrections in an analysis compared to a PSM.

\subsection{Applications}

Ashtekar variables only exist in three and four dimensions, such that an immediate loop quantization is possible only in those cases or in models which are obtained from them by symmetry reduction. With the formulation of general dilaton gravity models in spherically 
symmetric Ashtekar variables, however, we are now in a position to extend the loop quantization to arbitrary dilaton potentials: The Gauss and diffeomorphism constraint remain unaffected; in the Hamiltonian constraint, we simply insert the appropriate operator quantizing the given dilaton potential $V(\phi)$ in the spherically symmetric constraint operator; this will only change the coefficient $\hat{C}_{0}$, (66) , in a straightforward way: Instead of $1-\Gamma_{\varphi}^{2}$ we

then have $-V\left(E^{x}\right) \sqrt{\left|E^{x}\right|}-\Gamma_{\varphi}^{2}$, where $E^{x}$ in a triad representation will simply be replaced by $\frac{1}{2} \gamma \ell_{\mathrm{P}}^{2}\left(k_{+}+k_{-}\right)$after quantization. All the other coefficients in the difference equation remain unchanged, and so does the conclusion about the absence of singularities. Thus, all dilaton gravity models are singularity-free in a loop quantization.

This allows several specific applications. First, we can choose a linear dilaton potential $V(\phi) \propto \phi$, which provides a loop quantization of $B F$-theory. As one can see, the quantization does not simplify considerably in this case because most terms of the Hamiltonian constraint remain unchanged compared to spherically symmetric gravity. This is quite unexpected given that $B F$-theory can be quantized rather easily in different formulations. However, transformations between Ashtekar-type variables and variables which allow simple quantizations are non-trivial. Their quantizations can thus differ considerably. The $B F$-case of PSMs, quantized in Ashtekar variables as obtained here, can provide an interesting model for a quantization of a simple classical theory, quantized using techniques and basic objects as they apply to full gravity.

Secondly, we can provide loop quantizations of spherically symmetric models in arbitrary $D$ space-time dimensions. Here, we insert quantizations of the corresponding potentials $V(\phi) \propto \phi^{d(D)}$ which $d(D)=-1 /(D-2)$ from (13). Even though loop quantizations of general $D$-dimensional theories with $D>4$ have not been performed, at least non-rotating black holes can be studied by these models and compared with results from alternative quantizations in higher dimensions.

\section{Inverse triad quantization as a consistent deforma- tion}

We are now ready to introduce a specific type of quantum corrections to the classical models considered so far. This type of corrections results from quantizations of inverse powers of triad components in the loop formulation, as they appear in the Hamiltonian constraint. Densitized triads in loop quantum gravity are quantized by flux operators which have discrete spectra containing zero as an eigenvalue. Thus, they do not possess densely defined inverse operators. Nevertheless, as sketched before one can quantize inverse densitized triad components in a well-defined way [33], providing densely defined quantum constraint operators. For small values of the triad components near the classical divergence at zero, however, expectation values of the inverse triad operators in coherent states differ from the classical expression of the inverse. This deviation is captured by introducing a quantum correction function in terms of the constraint where inverse triad components appear via properties of triad operators in the loop representation. This type of correction 
is directly related to the underlying discreteness of geometric spectra.

Specifically, we have an inverse $E^{x}$ multiplying all terms in the Hamiltonian constraint (35) in Ashtekar variables. A quantum correction function $f$ is thus introduced into the Hamiltonian constraint as

$$
H_{\text {grav }}^{Q}[N]=\int d x N f \tilde{\mathcal{H}}_{\text {grav }}
$$

where $H_{\text {grav }}[N]=\int d x N \tilde{\mathcal{H}}_{\text {grav }}$ is the classical Hamiltonian (35). (See [29] for specific examples of correction functions.) The Gauss and diffeomorphism constraints remain unaltered since they do not contain inverse triad components.

Assuming that $f$ depends only on the densitized triad components $E^{x}$ and $E^{\varphi}$ (but not on their spatial derivatives and not on connection components) the full constraint algebra is

$$
\begin{gathered}
\left\{G_{\text {grav }}\left[\lambda_{1}\right], G_{\text {grav }}\left[\lambda_{2}\right]\right\}=0 \\
\left\{G_{\text {grav }}[\lambda], D_{\text {grav }}\left[N^{x}\right]\right\}=-G_{\text {grav }}\left[\mathcal{L}_{N^{x}} \lambda\right]=-G_{\text {grav }}\left[N^{x} \lambda^{\prime}\right] \\
\left\{G_{\text {grav }}[\lambda], H_{\text {grav }}^{Q}[N]\right\}=0 \\
\left\{D_{\text {grav }}\left[N^{x}\right], D_{\text {grav }}\left[M^{x}\right]\right\}=D_{\text {grav }}[[\bar{N}, \bar{M}]]=D_{\text {grav }}\left[N^{x} M^{x \prime}-M^{x} N^{x \prime}\right] \\
\left\{H_{\text {grav }}^{Q}[N], D_{\text {grav }}\left[N^{x}\right]\right\}=-H_{\text {grav }}^{Q}\left[N^{x} N^{\prime}-\frac{1}{f} \frac{\partial f}{\partial E^{\varphi}} E^{\varphi} N N^{x \prime}\right] \\
\left\{H_{\text {grav }}^{Q}[N], H_{\text {grav }}^{Q}[M]\right\}=D_{\text {grav }}\left[f^{2} \frac{E^{x}}{E^{\varphi} 2}\left(N M^{\prime}-M N^{\prime}\right)\right]+G_{\text {grav }}\left[f^{2} \frac{E^{x}}{E^{\varphi} 2}\left(N M^{\prime}-M N^{\prime}\right) \eta^{\prime}\right] .
\end{gathered}
$$

Thus, any correction of this form provides a first-class algebra, even though coefficients in the algebra are corrected compared to the classical case. We thus have a consistent deformation of the classical theory where the number of all gauge symmetries is preserved (even though the algebra does change). Note that $H_{\text {grav }}^{Q}$ transforms as a scalar only if $f$ is independent of $E^{\varphi}$ since $E^{\varphi}$ is the only quantity of density weight one. However, the vacuum algebra is first class even if $f$ does depend on $E^{\varphi}$. So far, this result is not surprising because the correction function $f$ simply multiplies the total Hamiltonian constraint and could thus be absorbed in the lapse function. (This by itself could change observable properties, as also discussed in [34, because it would still be the classical lapse function which enters the space-time metric (36) while the lapse function entering the Hamiltonian would be corrected by $f$. However, as far as consistency of the deformation is concerned, the vacuum case is rather trivial.)

The situation changes if we add matter terms by coupling a two-dimensional Yang-Mills system as in Sec. 4,

$$
S_{\mathrm{YM}}=\int d t \int d x \mathcal{A}_{x I} \dot{\mathcal{E}}^{I}-\mathcal{A}_{t I} \tilde{\mathcal{G}}_{\mathrm{YM}}-N \tilde{\mathcal{H}}_{\mathrm{YM}}
$$

with Gauss constraint

$$
G_{\mathrm{YM}}\left[\Lambda_{I}\right]=\int d x \Lambda_{I}\left(\mathcal{E}^{I^{\prime \prime}}+c_{J K}^{I} \mathcal{A}_{J} \mathcal{E}_{K}\right)
$$


and Hamiltonian

$$
H_{\mathrm{YM}}[N]=\int d x N \zeta E^{\varphi} \mathcal{E}^{I} \mathcal{E}_{I}
$$

There is no inverse triad component in this Hamiltonian, which remains uncorrected under the effects studied here. Thus, $f$ can no longer be absorbed in $N$ for the total constraint. The quantum corrected gravity-Yang-Mills Hamiltonian is

$$
H^{Q}[N]=H_{\text {grav }}^{Q}[N]+H_{\mathrm{YM}}[N]
$$

resulting in the full constraint algebra

$$
\begin{gathered}
\left\{G_{\mathrm{YM}}\left[\Lambda_{I}\right], G_{\mathrm{YM}}\left[V_{J}\right]\right\}=-G_{\mathrm{YM}}\left[c^{I J K} \Lambda_{I} V_{J}\right] \\
\left\{G_{\mathrm{YM}}\left[\Lambda_{I}\right], G_{\mathrm{grav}}[\lambda]\right\}=\left\{G_{\mathrm{YM}}\left[\Lambda_{I}\right], D_{\mathrm{grav}}\left[N^{x}\right]\right\}=0 \\
\left\{H^{Q}[N], G_{\mathrm{grav}}[\lambda]\right\}=\left\{H^{Q}[N], G_{\mathrm{YM}}\left[\Lambda_{I}\right]\right\}=0 \\
\left\{H^{Q}[N], D_{\text {grav }}\left[N^{x}\right]\right\}=-H_{\mathrm{grav}}^{Q}\left[N^{x} N^{\prime}-\frac{1}{f} \frac{\partial f}{\partial E^{\varphi}} E^{\varphi} N N^{x \prime}\right] \\
-H_{\mathrm{YM}}\left[N^{x} N^{\prime}\right]-G_{\mathrm{YM}}\left[2 N N^{x} \zeta E^{\varphi} E_{I}\right] \\
\left\{H^{Q}[N], H^{Q}[M]\right\}=D_{\text {grav }}\left[f^{2} \frac{E^{x}}{E^{\varphi 2}}\left(N M^{\prime}-M N^{\prime}\right)\right]+G_{\mathrm{grav}}\left[f^{2} \frac{E^{x}}{E^{\varphi} 2}\left(N M^{\prime}-M N^{\prime}\right) \eta^{\prime}\right] .
\end{gathered}
$$

In contrast to the vaccum case, there is now a non-trivial condition for the correction function: Only when $f$ does not depend on $E^{\varphi}$ can all the terms in $\left\{H^{Q}[N], D_{\text {grav }}\left[N^{x}\right]\right\}$ be combined to constraints. The dependence on $E^{x}$, on the other hand, is unrestricted. Thus, quantum corrections due to the loop quantization can provide non-trivial consistent deformations.

On the other hand, it was proved in [13] that a consistent deformation of the PSM in the sense of [35] must always be a PSM with the same dimension. Since the corrected constraint algebras (68) and (69) remain first class, the number of gauge symmetries does indeed stay fixed. It must be possible to formulate the quantum corrected system as a PSM. However, the result that any consistent deformation of a PSM must again be a PSM, as it follows from a BRST analysis, is obtained for equivalence classes of theories up to field redefinitions. This does not provide a constructive procedure to determine a corresponding PSM formulation for a given consistent deformation, and thus further input is required.

Rewriting the PSM constraints in terms of the standard gravitational constraints by inverting (47), (48) and (49):

$$
\tilde{C}^{\mp}=\left[\frac{1}{2 e} \tilde{\mathcal{D}}_{\text {grav }} \mp \frac{p}{\sqrt{2}} \phi^{-\frac{1}{4}} \tilde{\mathcal{H}}-\frac{\omega_{x}}{2 e} \tilde{C}^{3} \pm\left(\frac{\tilde{C}^{3}}{2 e}\right)^{\prime}\right] \exp (\mp \alpha)
$$


(with $\tilde{\mathcal{H}}$ either $\tilde{\mathcal{H}}_{\text {grav }}$ or $\tilde{\mathcal{H}}_{\text {grav }}+\tilde{\mathcal{H}}_{\text {YM }}$ ) and susbstituting $\tilde{\mathcal{H}}_{\text {grav }}$ for the quantum corrected Hamiltonian $f \tilde{\mathcal{H}}_{\text {grav }}$, we obtain a deformation of the PSM. It must be possible to cast the anomaly free algebras (68) and (69) as a PSM of some form. Finding this form will provide an action formulation for the quantum corrected system, and thus a covariant interpretation of the quantum correction function.

Inserting the correction function $f$ directly in (70) gives explicitly, in terms of PSM variables,

$$
\tilde{C}_{\text {deformed }}^{\mp}=\tilde{C}^{\mp} \mp(f[\phi]-1) \frac{1}{2 G}\left[\frac{1}{2} V(\phi) e+\frac{Q^{e}}{2}\left(\omega_{x}+\alpha^{\prime}\right)+\left(\frac{\phi^{\prime}}{2 e}\right)^{\prime}\right] \exp (\mp \alpha)
$$

with $\tilde{C}^{\mp}$ as in (23) or (53). This is not yet of a form suitable for a PSM interpretation due to the extra terms involving e.g. derivatives of $\phi$ which cannot simply be put in the dilaton potential. (The potential must be a function on the target space, which cannot accomodate space-time derivatives.)

Instead, we can use the requirement of the PSM form to find the corresponding formulation. In the previous equation, we have simply taken the same combinations of loop variable constraints as in the classical case. But if the constraints are corrected, we may well have to use different combinations of the constraints, with corrected coefficients, to bring them in a PSM form. We thus change the coefficients in front of the gravity constraints on the right hand side of (70) so as to exactly cancel the unwanted terms depending on $\phi$-derivatives. For this there is a unique way up to a total factor: the coefficient of $\tilde{\mathcal{H}}=f \tilde{\mathcal{H}}_{\text {grav }}+\tilde{\mathcal{H}}_{\mathrm{YM}}$ in the combination $\tilde{C}^{\mp}$ of constraints must carry an extra factor of $1 / f(\phi)$. In this way, the $\phi$-derivatives cancel in the combination of constraints as they do classically. The system is then described by a Poisson sigma model with constraints

$$
\tilde{C}_{Q}^{\mp}=\left[\frac{1}{2 e} \tilde{\mathcal{D}}_{\text {grav }} \mp \frac{p}{\sqrt{2}} \phi^{-\frac{1}{4}}\left(\tilde{\mathcal{H}}_{\text {grav }}+\frac{1}{f[\phi]} \tilde{\mathcal{H}}_{\mathrm{YM}}\right)-\frac{\omega_{x}}{2 e} \tilde{C}^{3} \pm\left(\frac{\tilde{C}^{3}}{2 e}\right)^{\prime}\right] \exp (\mp \alpha) .
$$

Here, the correction function appears only in one place multiplying the Yang-Mills Hamiltonian. The correction is thus non-trivial and changes the coupling of Yang-Mills to gravity: We now have the effective potential

$$
\frac{1}{2} V(\phi)+4 G \frac{\zeta(\phi)}{f(\phi)} \mathcal{E}^{I} \mathcal{E}_{I}
$$

In these models, the arbitrariness of $\zeta$ (in a $\phi$-dependent way) is thus enough to account for our consistent deformations: the deformed Poisson sigma model for (52) is of the same type with $\zeta$ replaced by $\zeta / f$. This is in accordance with our condition for a consistent deformation derived from Eq. (69), namely that $f$ only depends on $E^{x}$ which is identified with the dilaton $\phi$. Any other dependence could not be combined with the Yang-Mills coupling function $\zeta(\phi)$. 


\section{Conclusions}

We have studied the canonical relation between 2-dimensional dilaton gravity, Poisson sigma models and spherically symmetric gravity in Ashtekar variables. This is of interest because Ashtekar variables allow a background independent quantization of the full theory, while other quantization methods have been applied to dilaton gravity in two dimensions, such as a rigorous path integral quantization. Moreover, Poisson sigma models allow an interpretation of their structure functions as defining Lie algebroid symmetries, generalizing the Lie algebra symmetries of systems with structure constants. Given the explicit canonical transformation to Ashtekar variables we have derived, one may ask whether an analogous reformulation as a Lie algebroid sigma model could exist in four dimensions. If this would be the case, the structure function issue of general relativity could be substantially simplified. Unfortunately, the canonical transformation uses several special features realized only in two dimensions. For instance, as shown by Eq. (46) we need to eliminate a second derivative of $E^{x}$ which appears in the Hamiltonian constraint in Ashtekar variables but not in the PSM constraints. This can be done in two dimensions by means of a spatial derivative of the Gauss constraint. In four dimensions, on the other hand, the Gauss constraint contains the total divergence of the triad, which cannot provide all terms needed to remove all second triad derivatives from the full Hamiltonian constraint.

As a side result, we have used some of our derivations to extend the loop quantization to spherically symmetric systems in arbitrary $D$ space-time dimensions. This extends the proofs of singularity-freedom of spherically symmetric loop quantizations to spherically symmetric systems in arbitrary dimensions.

While dilaton gravity in two dimensions has been quantized covariantly by path integral methods, loop quantum gravity is a canonical quantization. In this context, the consistency issue of the resulting quantum constrained system is probably the most important one in loop quantum gravity, whose analysis will tell whether the diverse effects studied in simple models can be viable and covariant in general. What our analysis of consistency in two dimensions has shown is that there is indeed room for non-trivial effects due to the quantization. Quantum corrections of the canonical quantization are then related to a covariant action, where effective couplings to the Yang-Mills ingredients arose. Similar effects have been studied in the full four space-time dimensions but with perturbative inhomogeneities [36], where consistency was also shown to be possible.

For further corrections existing in a loop quantization, consistency has not yet been demonstrated. Among those we especially highlight the general phenomenon of quantum back-reaction, which implies that moments of a state such as fluctuations and correlations influence the dynamical behavior of expectation values. If this is included, new quantum degrees of freedom arise in an effective theory. In our context, a consistent deformation of this type will provide a higher-dimensional target space of the Poisson sigma model. Since the number of fields changes, the rigidity proofs for consistent deformations of PSMs no longer apply. Such effective theories could even generate new algebroid sigma models beyond PSMs, e.g. of the forms introduced in [20, 21]. 


\section{Acknowledgements}

We thank Daniel Grumiller and Thomas Strobl for discussions and comments. Some of the derivations described here were started while M.B. visited the Erwin-Schrödinger-Institute, Vienna, during the program "Poisson Sigma Models, Lie Algebroids, Deformations, and Higher Analogues," whose support is gratefully acknowledged. This work was supported in part by NSF grants PHY0653127 and 0748336.

\section{A Canonical transformation}

Here, we explicitly compute the canonical relation between Poisson sigma models and Ashtekar variables. The equation $\left\{Q^{e}, e\right\}=2 G$ gives

$$
-\gamma \frac{p s E^{\varphi}}{4\left|E^{x}\right|^{\frac{5}{4}}} \frac{\delta Q^{e}}{\delta A_{x}}+\frac{p}{2\left|E^{x}\right|^{\frac{1}{4}}} \frac{\delta Q^{e}}{\delta K_{\varphi}}=\sqrt{2}
$$

and $\left\{Q^{e}, \phi\right\}=0$ reads $\delta Q^{e} / \delta A_{x}=0$. Together they imply:

$$
Q^{e}=p 2 \sqrt{2} \|\left. E^{x}\right|^{\frac{1}{4}} K_{\varphi}+\tilde{Q}^{e}\left[E^{x}, E^{\varphi}, P^{\eta}, \eta\right]
$$

for arbitrary function $\tilde{Q}^{e}\left[E^{x}, E^{\varphi}, P^{\eta}, \eta\right]$.

Equations $\left\{Q^{\alpha}, e\right\}=0$ and $\left\{Q^{\alpha}, \phi\right\}=0$ give, respectively,

$$
-\gamma \frac{s E^{\varphi}}{4\left|E^{x}\right|^{\frac{5}{4}}} \frac{\delta Q^{\alpha}}{\delta A_{x}}+\frac{1}{2\left|E^{x}\right|^{\frac{1}{4}}} \frac{\delta Q^{\alpha}}{\delta K_{\varphi}}=0 \quad, \quad \frac{\delta Q^{\alpha}}{\delta A_{x}}=0
$$

which taken together imply

$$
Q^{\alpha}=Q^{\alpha}\left[E^{x}, E^{\varphi}, P^{\eta}, \eta\right]
$$

The equation $\left\{\phi, \omega_{x}\right\}=2 G$ just implies

$$
-\gamma \frac{s}{2} \frac{\delta \omega_{x}}{\delta A_{x}}=\frac{1}{2}
$$

and $\left\{e, \omega_{x}\right\}=0$ is equivalent to

$$
\frac{\delta \omega_{x}}{\delta K_{\varphi}}=\gamma \frac{s E^{\varphi}}{2\left|E^{x}\right|} \frac{\delta \omega_{x}}{\delta A_{x}}
$$

These two equations give the dependence of $\omega_{x}$ on $K_{x}$ and $K_{\varphi}$ :

$$
\omega_{x}=-\frac{s A_{x}}{\gamma}-\frac{E^{\varphi}}{2\left|E^{x}\right|} K_{\varphi}+\tilde{\omega}_{x}\left[E^{x}, E^{\varphi}, P^{\eta}, \eta\right]
$$

which fixes the sign ambiguity in (39) and gives

$$
\tilde{\omega}_{x}=-\frac{s \eta^{\prime}}{\gamma}-\alpha^{\prime}
$$


Equations $\{\phi, \alpha\}=0$ and $\{e, \alpha\}=0$ are, respectively,

$$
\frac{\delta \alpha}{\delta A_{x}}=0 \quad, \quad \frac{\delta \alpha}{\delta K_{\varphi}}=\gamma \frac{s E^{\varphi}}{2\left|E^{x}\right|} \frac{\delta \alpha}{\delta A_{x}} .
$$

Thus,

$$
\alpha=\alpha\left[E^{x}, E^{\varphi}, P^{\eta}, \eta\right]
$$

The remaining six equations $\left\{Q^{\alpha}, \alpha\right\}=2 G$ and $\left\{Q^{e}, \alpha\right\}=\left\{Q^{e}, Q^{\alpha}\right\}=\left\{Q^{e}, \omega_{x}\right\}=$ $\left\{Q^{\alpha}, \omega_{x}\right\}=\left\{\alpha, \omega_{x}\right\}=0$ are

$$
\begin{aligned}
\frac{\delta Q^{\alpha}}{\delta \eta} \frac{\delta \alpha}{\delta P^{\eta}}-\frac{\delta Q^{\alpha}}{\delta P^{\eta}} \frac{\delta \alpha}{\delta \eta} & =\frac{1}{\gamma} \\
\frac{\delta \tilde{Q}^{e}}{\delta \eta} \frac{\delta \alpha}{\delta P^{\eta}}-\frac{\delta \tilde{Q}^{e}}{\delta P^{\eta}} \frac{\delta \alpha}{\delta \eta} & =-p \frac{\sqrt{2}\left|E^{x}\right|^{\frac{1}{4}}}{\gamma} \frac{\delta \alpha}{\delta E^{\varphi}} \\
\frac{\delta \tilde{Q}^{e}}{\delta \eta} \frac{\delta Q^{\alpha}}{\delta P^{\eta}}-\frac{\delta \tilde{Q}^{e}}{\delta P^{\eta}} \frac{\delta Q^{\alpha}}{\delta \eta}- & =-p \frac{\sqrt{2}\left|E^{x}\right|^{\frac{1}{4}}}{\gamma} \frac{\delta Q^{\alpha}}{\delta E^{\varphi}} \\
\frac{\delta \tilde{Q}^{e}}{\delta \eta} \frac{\delta \tilde{\omega}_{x}}{\delta P^{\eta}}-\frac{\delta \tilde{Q}^{e}}{\delta P^{\eta}} \frac{\delta \tilde{\omega}_{x}}{\delta \eta} & =-\frac{1}{2 \gamma}\left(2 s \frac{\delta \tilde{Q}^{e}}{\delta E^{x}}+\frac{E^{\varphi}}{2\left|E^{x}\right|} \frac{\delta \tilde{Q}^{e}}{\delta E^{\varphi}}+p 2 \sqrt{2}\left|E^{x}\right|^{\frac{1}{4}} \frac{\delta \tilde{\omega}_{x}}{\delta E^{\varphi}}\right) \\
\frac{\delta Q^{\alpha}}{\delta \eta} \frac{\delta \tilde{\omega}_{x}}{\delta P^{\eta}}-\frac{\delta Q^{\alpha}}{\delta P^{\eta}} \frac{\delta \tilde{\omega}_{x}}{\delta \eta} & =-\frac{1}{2 \gamma}\left(2 s \frac{\delta Q^{\alpha}}{\delta E^{x}}+\frac{E^{\varphi}}{2\left|E^{x}\right|} \frac{\delta Q^{\alpha}}{\delta E^{\varphi}}\right) \\
\frac{\delta \alpha}{\delta \eta} \frac{\delta \tilde{\omega}_{x}}{\delta P^{\eta}}-\frac{\delta \alpha}{\delta P^{\eta}} \frac{\delta \tilde{\omega}_{x}}{\delta \eta} & =-\frac{1}{2 \gamma}\left(2 s \frac{\delta \alpha}{\delta E^{x}}+\frac{E^{\varphi}}{2\left|E^{x}\right|} \frac{\delta \alpha}{\delta E^{\varphi}}\right) .
\end{aligned}
$$

To try to find a solution to this system of equations we assume $\tilde{Q}^{e}$ to be independent of $P^{\eta}$ and $\eta$. Then (79) and (801) imply that $\alpha$ and $Q^{\alpha}$ are independent of $E^{\varphi}$, and we are left with equation (78) and

$$
\begin{gathered}
2 s \frac{\delta \tilde{Q}^{e}}{\delta E^{x}}+\frac{E^{\varphi}}{2\left|E^{x}\right|} \frac{\delta \tilde{Q}^{e}}{\delta E^{\varphi}}+p 2 \sqrt{2}\left|E^{x}\right|^{\frac{1}{4}} \frac{\delta \tilde{\omega}_{x}}{\delta E^{\varphi}}=0 \\
\frac{\delta Q^{\alpha}}{\delta \eta} \frac{\delta \tilde{\omega}_{x}}{\delta P^{\eta}}-\frac{\delta Q^{\alpha}}{\delta P^{\eta}} \frac{\delta \tilde{\omega}_{x}}{\delta \eta}=-\frac{s}{\gamma} \frac{\delta Q^{\alpha}}{\delta E^{x}} \\
\frac{\delta \alpha}{\delta \eta} \frac{\delta \tilde{\omega}_{x}}{\delta P^{\eta}}-\frac{\delta \alpha}{\delta P^{\eta}} \frac{\delta \tilde{\omega}_{x}}{\delta \eta}=-\frac{s}{\gamma} \frac{\delta \alpha}{\delta E^{x}} .
\end{gathered}
$$

Using (76) which implies $\frac{\delta \tilde{\omega}_{x}}{\delta E^{\varphi}}=0$, the equation for $\tilde{Q}^{e}$ is generically solved for $\tilde{Q}^{e}\left[E^{x}, E^{\varphi}\right]=$ $h\left(E^{\varphi} / \mid E^{x} \frac{1}{4}\right)$ with $h$ a function of one variable. If we further require that $C^{3}$ reproduce the Gauss constraint (33), $C^{3}=k G_{\text {grav }}$ for an arbitrary constant $k$, we find the solution giving rise to the canonical transformation (42).

\section{B Dilaton gravity with torsion}

For completeness, we summarize here the constructions necessary in the presence of torsion. This will not change the main results of the paper. 


\section{B.1 Generalized Dilaton Gravity and comparison of metrics}

Using the definitions 12 ]

$$
\phi:=\Phi^{2} \quad, \quad g_{\mu \nu}:=\mathbf{g}_{\mu \nu} \quad, \quad \mathbf{U}(\phi):=-\frac{1}{2 \phi} \quad, \quad \mathbf{V}(\phi):=1
$$

instead of (15), the spherically symmetric reduced $2 \mathrm{~d}$ action (12) is reexpressed as the generalized 2d Dilaton action

$$
S_{\text {dilaton }}=\frac{1}{2 G} \int d^{2} x \sqrt{-g}\left(\frac{1}{2} \phi R+W\left(-(\nabla \phi)^{2}, \phi\right)\right) .
$$

This in turn is equivalent to a general $2 \mathrm{~d}$ gravity action with torsion:

$$
S=-\frac{1}{2 G} \int_{M} \phi d \omega-W\left(X^{a} X_{a}, \phi\right) \varepsilon+X_{a} D e^{a}
$$

with

$$
W\left(X^{a} X_{a}, \phi\right):=\mathbf{U}(\phi) \frac{X^{a} X_{a}}{2}+\mathbf{V}(\phi)
$$

and $R=2 * d \bar{\omega}$ the curvature for the torsion free part of the spin connection $\omega=\bar{\omega}+e_{a} \frac{\partial W}{\partial X_{a}}$. There is no conformal transformation, so the metric $g$ represents the physical metric in this approach.

The Poisson sigma model in this case is determined by the more general Poisson bivector

$$
\mathcal{P}^{i j}=\left(\begin{array}{ccc}
0 & W & -X^{-} \\
-W & 0 & X^{+} \\
X^{-} & -X^{+} & 0
\end{array}\right)
$$

so that in the constraints (23), $V / 2$ is replaced by $-W$ :

$$
\tilde{C}^{\mp}=\frac{1}{2 G}\left[\left(\frac{e Q^{e} \pm Q^{\alpha}}{2 e}\right)^{\prime} \mp\left(\frac{e Q^{e} \pm Q^{\alpha}}{2 e}\right)\left(\omega_{x}+\alpha^{\prime}\right) \pm W\left(2 X^{+} X^{-}, \phi\right) e\right] \exp (\mp \alpha)
$$

and from (22)

$$
2 X^{+} X^{-}=2\left(\frac{e Q^{e}-Q^{\alpha}}{2 e}\right)\left(\frac{e Q^{e}+Q^{\alpha}}{2 e}\right) .
$$

Comparison of the metrics now yields different values for $\phi$ and the dyads (38) which get an extra factor of $\left|E^{x}\right|^{-\frac{1}{4}}$ :

$$
\begin{aligned}
\phi & =\left|E^{x}\right| \\
e_{x}^{+} & =p \frac{E^{\varphi}}{\sqrt{2}\left|E^{x}\right|^{\frac{1}{2}}} \exp \alpha \quad, \quad e_{x}^{-}=p \frac{E^{\varphi}}{\sqrt{2}\left|E^{x}\right|^{\frac{1}{2}}} \exp (-\alpha) \\
e_{t}^{+} & =p \frac{N^{x} E^{\varphi} \pm N\left|E^{x}\right|^{\frac{1}{2}}}{\sqrt{2}\left|E^{x}\right|^{\frac{1}{2}}} \exp \alpha \quad, \quad e_{t}^{-}=p \frac{1}{\sqrt{2}\left|E^{x}\right|^{\frac{1}{2}}}\left(\frac{-N^{2}\left|E^{x}\right|+N^{x} E^{\varphi} 2}{N^{x} E^{\varphi} \pm N\left|E^{x}\right|^{\frac{1}{2}}}\right) \exp (-\alpha)
\end{aligned}
$$


The dependence of the Lagrange multipliers $X^{ \pm}$in terms of $\left(E^{x}, E^{\varphi}, K_{x}, K_{\varphi}\right)$ (as in equations (40141)) consequently gets an extra factor of $\left|E^{x}\right|^{\frac{1}{4}}$ :

$$
\begin{aligned}
& X^{-}=p \sqrt{2}\left|E^{x}\right|^{\frac{1}{2}}\left(-s \frac{E^{x \prime}}{2 E^{\varphi}} \mp K_{\varphi}\right) \exp (-\alpha) \\
& X^{+}=p \sqrt{2}\left|E^{x}\right|^{\frac{1}{2}}\left(s \frac{E^{x \prime}}{2 E^{\varphi}} \mp K_{\varphi}\right) \exp (\alpha)
\end{aligned}
$$

The torsion free part of the spin connection $\omega_{x}$ is $\bar{\omega}_{x}= \pm K_{x}-\alpha^{\prime}$, and the torsion dependent part $e_{x a} \frac{\partial W}{\partial X_{a}}=\mathbf{U}(\phi) e_{x a} X^{a}= \pm 2 \mathbf{U}\left(E^{x}\right) E^{\varphi} K_{\varphi}$, so

$$
\omega_{x}= \pm s K_{x} \mp 2 \mathbf{U}\left(E^{x}\right) E^{\varphi} K_{\varphi}-\alpha^{\prime}
$$

\section{B.2 Canonical transformation}

The Poisson bracket relations

$$
\left\{Q^{e}(x), e(y)\right\}=\left\{Q^{\alpha}(x), \alpha(y)\right\}=\left\{\phi(x), \omega_{x}(y)\right\}=2 G \delta(x, y)
$$

give the following functional dependence of $\left(Q^{e}, Q^{\alpha}, \phi ; e, \alpha, \omega_{x}\right)$ in terms of $\left(E^{x}, E^{\varphi}, P^{\eta} ; K_{x}, K_{\varphi}, \eta\right)$ :

$$
\begin{aligned}
\phi=\left|E^{x}\right| & , \quad e=\frac{E^{\varphi}}{\sqrt{2}\left|E^{x}\right|^{\frac{1}{2}}} \\
\omega_{x}=-s \frac{A_{x}}{\gamma}-\frac{E^{\varphi}}{\left|E^{x}\right|} K_{\varphi}+\tilde{\omega}_{x}\left[E^{x}, E^{\varphi}, P^{\eta}, \eta\right] & , \quad Q^{e}=p \sqrt{2}\left|E^{x}\right|^{\frac{1}{2}} K_{\varphi}+\tilde{Q}^{e}\left[E^{x}, E^{\varphi}, P^{\eta}, \eta\right] \\
Q^{\alpha}=Q^{\alpha}\left[E^{x}, E^{\varphi}, P^{\eta}, \eta\right] & , \quad \alpha=\alpha\left[E^{x}, E^{\varphi}, P^{\eta}, \eta\right]
\end{aligned}
$$

and the following differential equations analogous to (78), (779), (801), (81), (82), (83):

$$
\begin{aligned}
& \frac{\delta Q^{\alpha}}{\delta \eta} \frac{\delta \alpha}{\delta P^{\eta}}-\frac{\delta Q^{\alpha}}{\delta P^{\eta}} \frac{\delta \alpha}{\delta \eta}=\frac{1}{\gamma} \\
& \frac{\delta \tilde{Q}^{e}}{\delta \eta} \frac{\delta \alpha}{\delta P^{\eta}}-\frac{\delta \tilde{Q}^{e}}{\delta P^{\eta}} \frac{\delta \alpha}{\delta \eta}=-p \frac{\sqrt{2}\left|E^{x}\right|^{\frac{1}{2}}}{\gamma} \frac{\delta \alpha}{\delta E^{\varphi}} \\
& \frac{\delta \tilde{Q}^{e}}{\delta \eta} \frac{\delta Q^{\alpha}}{\delta P^{\eta}}-\frac{\delta \tilde{Q}^{e}}{\delta P^{\eta}} \frac{\delta Q^{\alpha}}{\delta \eta}=-p \frac{\sqrt{2}\left|E^{x}\right|^{\frac{1}{2}}}{\gamma} \frac{\delta Q^{\alpha}}{\delta E^{\varphi}} \\
& \frac{\delta \tilde{Q}^{e}}{\delta \eta} \frac{\delta \tilde{\omega}_{x}}{\delta P^{\eta}}-\frac{\delta \tilde{Q}^{e}}{\delta P^{\eta}} \frac{\delta \tilde{\omega}_{x}}{\delta \eta}=-\frac{1}{2 \gamma}\left(2 s \frac{\delta \tilde{Q}^{e}}{\delta E^{x}}+\frac{E^{\varphi}}{\left|E^{x}\right|} \frac{\delta \tilde{Q}^{e}}{\delta E^{\varphi}}+p 2 \sqrt{2}\left|E^{x}\right|^{\frac{1}{2}} \frac{\delta \tilde{\omega}_{x}}{\delta E^{\varphi}}\right) \\
& \frac{\delta Q^{\alpha}}{\delta \eta} \frac{\delta \tilde{\omega}_{x}}{\delta P^{\eta}}-\frac{\delta Q^{\alpha}}{\delta P^{\eta}} \frac{\delta \tilde{\omega}_{x}}{\delta \eta}=-\frac{1}{2 \gamma}\left(2 s \frac{\delta Q^{\alpha}}{\delta E^{x}}+\frac{E^{\varphi}}{\left|E^{x}\right|} \frac{\delta Q^{\alpha}}{\delta E^{\varphi}}\right) \\
& \frac{\delta \alpha}{\delta \eta} \frac{\delta \tilde{\omega}_{x}}{\delta P^{\eta}}-\frac{\delta \alpha}{\delta P^{\eta}} \frac{\delta \tilde{\omega}_{x}}{\delta \eta}=-\frac{1}{2 \gamma}\left(2 s \frac{\delta \alpha}{\delta E^{x}}+\frac{E^{\varphi}}{\left|E^{x}\right|} \frac{\delta \alpha}{\delta E^{\varphi}}\right)
\end{aligned}
$$


Already, from the form of $\omega_{x}$ in (88) and in (87) we see that a canonical transformation is only consistent for $\mathbf{U}(\phi)=-\frac{1}{2 \phi}$. So we only have freedom to change the functional form of $\mathbf{V}(\phi)$ if we try to generalize to other models (just as in the conformal approach).

Attempting to solve these differential equations by assuming $\tilde{Q}^{e}=0$ and requiring $C^{3}$ to reproduce the Gauss constraint as before gives the transformation

$$
\begin{aligned}
& Q^{e}=p 2 \sqrt{2}\left|E^{x}\right|^{\frac{1}{2}} K_{\varphi}+h\left[\left|E^{x}\right|^{-\frac{1}{2}} E^{\varphi}\right], e=p \frac{E^{\varphi}}{\sqrt{2}\left|E^{x}\right|^{\frac{1}{2}}} \\
& \phi=\left|E^{x}\right|, \quad \omega_{x}=-s K_{x}-\frac{E^{\varphi}}{\left|E^{x}\right|} K_{\varphi}+\frac{1}{k} \eta^{\prime} \\
& Q^{\alpha}=\frac{k}{\gamma} P^{\eta}+\left(\frac{k-s \gamma}{\gamma}\right) E^{x \prime}, \quad \alpha=-\frac{1}{k} \eta
\end{aligned}
$$

and its inverse

$$
\begin{array}{rlrl}
E^{x}=s \phi \quad, \quad E^{\varphi} & =p \sqrt{2} \phi^{\frac{1}{2}} e \\
K_{x}=-s\left(\omega_{x}+\alpha^{\prime}+\frac{e}{2 \phi}\left(Q^{e}-h\right)\right) \quad, \quad K_{\varphi} & =p \frac{\left(Q^{e}-h\right)}{2 \sqrt{2} \phi^{\frac{1}{2}}} \\
\eta=-k \alpha & , \quad P^{\eta} & =\frac{\gamma}{k} Q^{\alpha}+\left(\frac{\gamma-s k}{k}\right) \phi^{\prime}
\end{array}
$$

where again, $s=\operatorname{sign}\left(E^{x}\right), k$ is an arbitrary constant, and $h$ an arbitrary function of one variable.

\section{B.3 Constraints}

Again, for $h=0$ we have

$$
C^{3}[\lambda]=k G_{\text {grav }}[\lambda] \quad, \quad D\left[N^{x}\right]=D_{\text {grav }}\left[N^{x}\right]
$$

and the remaining linear combination is

$$
\begin{aligned}
& C^{+}[N \exp (-\alpha)]- C^{-}[N \exp (\alpha)]=\frac{1}{2 G} \int d x N\left(-Q^{e}\left(\omega_{x}+\alpha^{\prime}\right)-\left(\frac{Q^{\alpha}}{e}\right)^{\prime}-2 W e\right) \\
&=\frac{p \sqrt{2}}{2 G} \int d x N\left[-\left|E^{x}\right|^{-\frac{1}{2}} K_{\varphi}^{2} E^{\varphi}-2 s\left|E^{x}\right|^{\frac{1}{2}} K_{x} K_{\varphi}-\left|E^{x}\right|^{-\frac{1}{2}} E^{\varphi} \mathbf{V}\right. \\
&\left.+\frac{\left|E^{x}\right|^{-\frac{1}{2}} E^{x \prime 2}}{4 E^{\varphi}}-\frac{s\left|E^{x}\right|^{\frac{1}{2}} E^{x \prime} E^{\varphi \prime}}{E^{\varphi} 2}+\frac{s\left|E^{x}\right|^{\frac{1}{2}} E^{x \prime \prime}}{E^{\varphi}}\right] \\
&+\frac{k}{2 G \gamma} \int d x N\left[\frac{s E^{x \prime}}{2\left|E^{x}\right|^{\frac{1}{2}} E^{\varphi}}\left(E^{x \prime}+P^{\eta}\right)-\frac{k}{4 \gamma \mid E^{x \mid \frac{1}{2}} E^{\varphi}}\left(E^{x \prime}+P^{\eta}\right)^{2}\right. \\
&\left.-\left(\frac{\left|E^{x}\right|^{\frac{1}{2}}}{E^{\varphi}}\left(E^{x \prime}+P^{\eta}\right)\right)^{\prime}\right] .
\end{aligned}
$$


For $\mathbf{V}(\phi)=1$, this gives

$$
\begin{aligned}
& C^{+}[N \exp (-\alpha)]-C^{-}[N \exp (\alpha)]=p \sqrt{2} H_{\text {grav }}[N] \\
& +\frac{k}{2 G \gamma} \int d x N\left[\frac{s E^{x \prime}}{2 \mid E^{x \mid \frac{1}{2}} E^{\varphi}}\left(E^{x \prime}+P^{\eta}\right)-\frac{k}{4 \gamma\left|E^{x}\right|^{\frac{1}{2}} E^{\varphi}}\left(E^{x \prime}+P^{\eta}\right)^{2}\right. \\
& \left.-\left(\frac{\left|E^{x}\right|^{\frac{1}{2}}}{E^{\varphi}}\left(E^{x \prime}+P^{\eta}\right)\right)^{\prime}\right]
\end{aligned}
$$

In summary,

$$
\begin{aligned}
\tilde{C}^{3} & =k \tilde{\mathcal{G}}_{\text {grav }} \\
e \exp (\alpha) \tilde{C}^{-}+e \exp (-\alpha) \tilde{C}^{+}+\omega_{x} \tilde{C}^{3} & =\tilde{\mathcal{D}}_{\text {grav }} \\
-\exp (\alpha) \tilde{C}^{-}+\exp (-\alpha) \tilde{C}^{+}-\frac{\phi^{\prime}-G \tilde{C}^{3}}{2 e \phi} \tilde{C}^{3}+\left(\frac{\tilde{C}^{3}}{e}\right)^{\prime} & =p \sqrt{2} \tilde{\mathcal{H}}_{\text {grav }} .
\end{aligned}
$$

\section{References}

[1] C. Rovelli, Quantum Gravity, Cambridge University Press, Cambridge, UK, 2004

[2] A. Ashtekar and J. Lewandowski, Background independent quantum gravity: A status report, Class. Quantum Grav. 21 (2004) R53-R152, gr-qc/0404018

[3] T. Thiemann, Introduction to Modern Canonical Quantum General Relativity, Cambridge University Press, Cambridge, UK, 2007, gr-qc/0110034

[4] T. Thiemann, Quantum Spin Dynamics (QSD), Class. Quantum Grav. 15 (1998) 839-873, gr-qc/9606089

[5] M. Bojowald, Inverse Scale Factor in Isotropic Quantum Geometry, Phys. Rev. D 64 (2001) 084018, gr-qc/0105067

[6] C. Rovelli and L. Smolin, Loop Space Representation of Quantum General Relativity, Nucl. Phys. B 331 (1990) 80-152

[7] M. Bojowald and A. Skirzewski, Effective Equations of Motion for Quantum Systems, Rev. Math. Phys. 18 (2006) 713-745, math-ph/0511043

[8] M. Bojowald and A. Skirzewski, Quantum Gravity and Higher Curvature Actions, Int. J. Geom. Meth. Mod. Phys. 4 (2007) 25-52, [hep-th/0606232], Proceedings of "Current Mathematical Topics in Gravitation and Cosmology" (42nd Karpacz Winter School of Theoretical Physics), Ed. Borowiec, A. and Francaviglia, M.

[9] M. Bojowald, Loop Quantum Cosmology, Living Rev. Relativity 11 (2008) 4, gr-qc/0601085, http://www.livingreviews .org/lrr-2008-4 
[10] M. Bojowald, Spherically Symmetric Quantum Geometry: States and Basic Operators, Class. Quantum Grav. 21 (2004) 3733-3753, gr-qc/0407017

[11] T. Strobl, Gravity in Two Spacetime Dimensions, Habilitation Thesis, hep-th/0011240

[12] D. Grumiller, W. Kummer, and D. V. Vassilevich, Dilaton Gravity in Two Dimensions, Phys. Rept. 369 (2002) 327-430, |hep-th/0204253

[13] K. I. Izawa, On nonlinear gauge theory from a deformation theory perspective, Prog. Theor. Phys. 103 (2000) 225, hep-th/9910133

[14] N. Ikeda, Two-Dimensional Gravity and Nonlinear Gauge Theory, Ann. Phys. 235 (1994) 435-464, hep-th/9312059

[15] P. Schaller and T. Strobl, Poisson Structure Induced (Topological) Field Theories, Mod. Phys. Lett. A 9 (1994) 3129-3136, hep-th/9405110

[16] N. Ikeda and K. I. Izawa, General form of dilaton gravity and nonlinear gauge theory, Prog. Theor. Phys. 90 (1993) 237, hep-th/9304012

[17] M. Bojowald, A. Kotov, and T. Strobl, Lie algebroid morphisms, Poisson Sigma Models, and off-shell closed gauge symmetries, J. Geom. Phys. 54 (2005) 400-426, math.DG/0406445

[18] M. Bojowald, H. A. Kastrup, F. Schramm, and T. Strobl, Group Theoretical Quantization of a Phase Space $S^{1} \times \mathbb{R}^{+}$and the Mass Spectrum of Schwarzschild Black Holes in D Space-Time Dimensions, Phys. Rev. D 62 (2000) 044026, [gr-qc/9906105]

[19] D. Grumiller, Quantum Dilaton Gravity in Two Dimensions with Matter, PhD thesis, TU Vienna, gr-qc/0105078

[20] T. Strobl, Algebroid Yang-Mills Theories, Phys. Rev. Lett. 93 (2004) 211601, hep-th/0406215

[21] A. Kotov, P. Schaller, and T. Strobl, Dirac Sigma Models, Commun. Math. Phys. 260 (2005) 455-480, hep-th/0411112

[22] A. Ashtekar, New Hamiltonian Formulation of General Relativity, Phys. Rev. D 36 (1987) 1587-1602

[23] J. F. Barbero G., Real Ashtekar Variables for Lorentzian Signature Space-Times, Phys. Rev. D 51 (1995) 5507-5510, gr-qc/9410014

[24] G. Immirzi, Real and Complex Connections for Canonical Gravity, Class. Quantum Grav. 14 (1997) L177-L181 
[25] T. Thiemann and H. A. Kastrup, Canonical Quantization of Spherically Symmetric Gravity in Ashtekar's Self-Dual Representation, Nucl. Phys. B 399 (1993) 211-258, gr-qc/9310012

[26] M. Bojowald and H. A. Kastrup, Symmetry Reduction for Quantized Diffeomorphism Invariant Theories of Connections, Class. Quantum Grav. 17 (2000) 3009-3043, hep-th/9907042

[27] M. Bojowald and R. Swiderski, Spherically Symmetric Quantum Geometry: Hamiltonian Constraint, Class. Quantum Grav. 23 (2006) 2129-2154, [gr-qc/0511108]

[28] M. Bojowald, Loop quantum cosmology and inhomogeneities, Gen. Rel. Grav. 38 (2006) 1771-1795, [gr-qc/0609034]

[29] M. Bojowald, T. Harada, and R. Tibrewala, Lemaitre-Tolman-Bondi collapse from the perspective of loop quantum gravity, Phys. Rev. D 78 (2008) 064057, arXiv:0806.2593

[30] M. Bojowald, Singularities and Quantum Gravity, AIP Conf. Proc. 910 (2007) 294333, gr-qc/0702144], Proceedings of the XIIth Brazilian School on Cosmology and Gravitation

[31] M. Bojowald, Non-singular black holes and degrees of freedom in quantum gravity, Phys. Rev. Lett. 95 (2005) 061301, gr-qc/0506128

[32] M. Bojowald, B. Sandhöfer, A. Skirzewski, and A. Tsobanjan, Effective constraints for quantum systems, arXiv:0804.3365

[33] T. Thiemann, QSD V: Quantum Gravity as the Natural Regulator of Matter Quantum Field Theories, Class. Quantum Grav. 15 (1998) 1281-1314, [gr-qc/9705019]

[34] M. Bojowald and G. Hossain, Quantum gravity corrections to gravitational wave dispersion, Phys. Rev. D 77 (2008) 023508, arXiv:0709.2365]

[35] G. Barnich and M. Henneaux, Consistent couplings between fields with a gauge freedom and deformations of the master equation, Phys. Lett. B 311 (1993) 123, hep-th/9304057

[36] M. Bojowald, G. Hossain, M. Kagan, and S. Shankaranarayanan, Anomaly freedom in perturbative loop quantum gravity, Phys. Rev. D 78 (2008) 063547, arXiv:0806.3929. 